Este trabajo ha obtenido el Accésit del Premio Estudios Financieros 2017 en la modalidad de Educación y Nuevas Tecnologías. El jurado ha estado compuesto por: don José Ignacio Baile Ayensa, don Julio Cabero Almenara, doña Trinidad Manzano Moreno, don Antonio Pastor Sanmillán, don Juan Pazos Sierra y don Ismael Sanz Labrador. (Los trabajos se presentan con seudónimo y la selección se efectúa garantizando el anonimato de los autores)

\title{
Determinantes de la experiencia de cliente en e-servicios: el caso de las universidades virtuales
}

\section{Extracto:}

Sumario

El auge de la universidad online plantea muchas cuestiones relacionadas con la competitividad y permanencia a largo plazo de este tipo de instituciones. Desde esta perspectiva, uno de los aspectos que puede contribuir a incrementar su competitividad a largo plazo es la experiencia de los clientes, en este caso, los estudiantes.

2. La experiencia del cliente en el contexto online

\section{Metodología}

4. Análisis de resultados

5. Conclusiones

6. Bibliografía

\section{Apéndices}

Teniendo como antecedentes estas ideas previas, este estudio analiza los factores determinantes de la experiencia de los clientes en los servicios electrónicos. En concreto, el objetivo consiste en definir y cuantificar la influencia que la calidad del sitio web y la marca universidad ejercen sobre la experiencia de los estudiantes de una universidad virtual. El análisis empírico realizado -utilizando PLS-SEM (partial least squares-structural equation modeling)- ha permitido poner de manifiesto que la marca de la universidad es el factor que más influye sobre la experiencia del estudiante. Por otro lado, se observa que la influencia ejercida por el entorno web donde se ofrece el servicio es menor. De las tres dimensiones identificadas, la calidad del sistema es la que ejerce una mayor influencia, seguida de la calidad de la relación, la cual viene moderada por el rol ejercido por el personal del staff universitario.

Palabras clave: experiencia de cliente, calidad de la web, valoración de la marca, entorno virtual de aprendizaje, universidad virtual.

\footnotetext{
A. Izquierdo Yusta, profesora titular de universidad (Universidad de Burgos).

${ }^{2}$ A. I. Jiménez Zarco, profesora agregada de la Universitat Oberta de Catalunya (UOC).

${ }^{3}$ I. González González, profesora del Área de Empresa de la Universidad Internacional de La Rioja (UNIR).

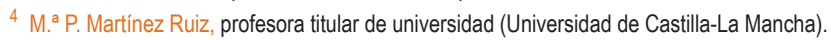




\section{Drivers of} e-services' consumer

\section{experience: the} case of online universities

\begin{abstract}
:
The proliferation of online universities has open many questions arise regarding the competitiveness and long-term permanence of this kind of institutions. From this perspective, one of the aspects that can contribute to increase their long-term competitiveness is the clients' experience, in this case, the students.
\end{abstract}

According the last ideas, this study analyses the key drivers of consumer experience in e-services. Particularly our goal is define and quantify the influence that website quality and university brand assess have on consumers experience, when he/she is a student of a virtual university. Using the PLS-SEM (partial least squaresstructural equation modeling) has enabled us to demonstrate how the university brand is the most influential factor in the student's experience. On the other hand, it is observed how the influence exerted by the web environment is lower where the service is offered. Of the three dimensions identified, the quality of the system is the one that exerts a greater influence, followed by the quality of the relationship, which is moderated by the role played by university staff.

Keywords: customer experience, website quality, brand assess, learning virtual environment, online university.

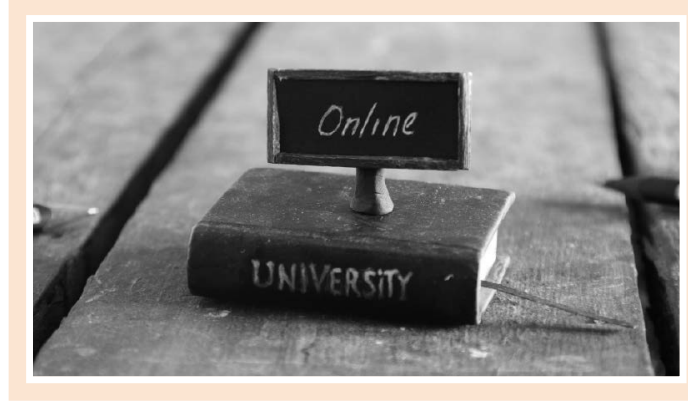

\section{INTRODUCCIÓN}

El uso intensivo de las denominadas «tecnologías de la información y la comunicación» (TIC) ha revolucionado la actividad universitaria. Sus efectos se hacen patentes tanto en el modo en el que se desarrolla su actividad como en la orientación estratégica que define su gestión. A nivel formativo, se observa el desarrollo de nuevos modelos en el proceso de enseñanza-aprendizaje, con la consiguiente redefinición de las figuras del profesor y del estudiante, así como de la relación establecida entre ellos (Elsharnouby, 2015; Pimienta, Barzola y Zurdo, 2016). Por otro lado, las TIC también han modificado el entorno en el que se desarrolla la relación, favoreciendo la aparición de entornos virtuales de aprendizaje, nuevos recursos docentes y productos formativos. Finalmente, la tecnología también ha hecho posible que el mercado potencial y la competencia que encuentran las universidades en sus entornos de referencia pueda ser calificada como global. Es por ello que desde comienzos de la pasada década se observa un cambio en los modelos estratégicos y de gestión desarrollados por las universidades. 
Con el fin de sobrevivir y consolidar sus posiciones en el mercado, las universidades han comenzado a adoptar una orientación hacia los stakeholders, donde particularmente el estudiante -cliente de los servicios académicos- se convierte en el centro de la actividad universitaria (Farrel et al., 2001; Greenley, Hooley y Rudd, 2005). Así, la creciente orientación al marketing en la educación ha llevado a que el estudio y la comprensión de la satisfacción del estudiante se convierta en un elemento fundamental en la gestión universitaria (Mateo, 2015). Dado que son los estudiantes los que permiten que las instituciones educativas existan y perduren en el tiempo, resulta clave lograr que queden satisfechos, lo que reducirá sus motivos para abandonar o cambiar de universidad.

Ahora bien, si tradicionalmente conseguir la satisfacción del cliente determinaba el diseño de las estrategias de marketing de las empresas, en la actualidad, el énfasis se centra en lograr vincular emocionalmente al cliente, residiendo la clave en la experiencia de este último. Al respecto, cabe señalar que algunos trabajos, como los de Rose et al. (2012), destacan la existencia de importantes diferencias entre los conceptos de «satisfacción» y «experiencia de cliente». La satisfacción presenta un mayor grado de objetividad y se relaciona directamente con el resultado, en cambio, la experiencia presenta un mayor grado de subjetividad y se relaciona tanto con el resultado obtenido como con el proceso que permite conseguirlo (Srivastava y Kaul, 2016). Así, un cliente puede estar satisfecho porque encontró lo que buscaba, pero pudo haber tenido una pésima experiencia en el proceso. Además, y lo que resulta fundamental para la empresa, la simple satisfacción no crea vínculos entre el cliente y la marca. Es especialmente en aquellas situaciones en las que el cliente se siente emocionalmente unido con la marca -por ejemplo, situaciones en las que se siente orgulloso o agradecido con esta- cuando los niveles de lealtad son elevados.

\section{En el diseño de las estrategias de marketing de las empresas, en la actualidad, el énfasis se centra en lograr vincular emocionalmente al cliente, residiendo la clave en la experiencia de este último}

Trasladar esta idea al ámbito universitario implica necesariamente considerar la experiencia del estudiante como uno de los determinantes del éxito de la institución universitaria. Además, en un contexto donde una parte importante de la actividad docente se desarrolla en entornos virtuales, las universidades deben comenzar a considerar de qué forma la experiencia del estudiante viene condicionada por el contexto donde se desarrolla el proceso de aprendizaje.

Atendiendo a esta situación, el presente trabajo analiza los determinantes de la experiencia del estudiante en un entorno universitario online. Para ello, se parte del concepto «experiencia de cliente», para posteriormente analizarlo en el contexto de los servicios universitarios online. El modelo propuesto por Schmitt y Zarantonello (2013) sirve de base para establecer que la calidad del aula virtual y la valoración de la marca constituyen los determinantes principales de la experiencia del estudiante universitario online. A fin de contrastar las hipótesis propuestas, se analiza una muestra de estudiantes de la UOC. Los resultados obtenidos confirman la validez del modelo, poniendo de manifiesto que los factores anteriormente señalados influyen de forma positiva en la experiencia del estudiante universitario.

\section{LA EXPERIENCIA DEL CLIENTE EN EL CONTEXTO ONLINE}

La vinculación emocional del cliente con la marca - o compañía- se ha convertido en uno de los principales indicadores del éxito empresarial (Rose et al., 2012). Smilansky (2009) señala que, actualmente, ofrecer productos o servicios de calidad ya no es suficiente para asegurar la competitividad e incluso la supervivencia de las empresas. Es más, ciertos trabajos han puesto en duda que obtener la satisfacción del cliente garantice su fidelidad (Leverin y Liljander, 2006; Kumar, Dalla Pozza y Ganesh, 2013) y, lo que es más importante, su recomendación (Dabholkar y Sheng, 2012). 
Las empresas compiten en un entorno complejo y rápidamente cambiante, y la actual crisis económica y de valores contribuye a acentuar esta situación. Hoy en día la mayoría de compañías se mueven en mercados maduros, altamente competitivos, con productos y servicios casi indiferenciados y optando por posicionamientos basados esencialmente en el precio. Los clientes son ahora más exigentes, están mejor informados y buscan productos personalizados. Todo ello lleva a que en términos estratégicos se esté produciendo un cambio de paradigma que lleva al cliente no solo a desear cubrir sus necesidades básicas, sino a querer elevar sus procesos de compra y consumo a un estadio superior, el de las emociones y experiencias (Schmitt, 2000).

Por otro lado, el desarrollo de las TIC no solo permite actuar en un nuevo contexto competitivo, sino que también ha abierto la puerta a un nuevo entorno de colaboración, de diálogo y de relación. En este nuevo escenario, el cliente desarrolla un papel más directo y activo en su relación con la marca (Gómez-Suárez, Martínez-Ruiz y Martínez-Caraballo, 2017). Además, esta relación puede ser continuada en el tiempo, de tal manera que el número de interacciones y experiencias obtenidas llegue a ser más elevado (Gentile, Spiller y Noci, 2007).

En aras de fortalecer la relación con sus clientes, la empresa no solo debe monitorizar todo el conjunto de experiencias, sino, lo que es más importante, identificar y gestionar los factores que las determinan con la finalidad de establecer políticas de marketing que permitan que la experiencia total del cliente sea positiva.

\subsection{El concepto de «experiencia de cliente»}

Meyer y Schwager (2007) definen la experiencia de cliente como la respuesta interna, integral y subjetiva que ofrece el cliente ante cualquier contacto directo o indirecto con la compañía o la marca. Su naturaleza psicológica se evidencia en los trabajos de Gentile, Spiller y Noci (2007), Frow y Payne (2007), Tynan y McKechnie (2009) o Zomerdijk y Voss (2011) al señalar que la experiencia del individuo viene determinada hasta por seis componentes diferentes:

\section{- Sensorial.}

- Emocional.

\section{La experiencia del cliente se origina en el conjunto de interacciones que tienen lugar entre un cliente $y$ un producto o servicio, una empresa o parte de su organización [...]. Es estrictamente personal y conlleva la implicación del cliente a diferentes niveles (racional, emocional, sensorial, físico y espiritual)}

- Cognitivo.

- Pragmático.

- Estilo de vida.

- Relacional.

En esta misma línea, Bigné-Alcañiz y Andreu-Simó (2004) reconocen la existencia de tres componentes de las emociones:

- Cognitivo.

- Emocional.

- Conductual.

Para nuestra investigación, proponemos una definición de la experiencia del cliente que tiene en cuenta las contribuciones científicas más relevantes. Específicamente, definimos el concepto de «experiencia de cliente» como una evolución del concepto de «relación entre la empresa y el cliente». La experiencia del cliente se origina en el conjunto de interacciones que tienen lugar entre un cliente y un producto o servicio, una empresa o parte de su organización, lo cual provoca una reacción (LaSalle y Britton, 2003; Shaw e Ivens, 2005). Esta experiencia es estrictamente personal y conlleva la implicación del cliente a diferentes niveles (racional, emocional, sensorial, físico y espiritual) (LaSalle y Britton, 2003; Schmitt, 2000), cuya evaluación depende de la comparación entre las expectativas del cliente y los estímulos procedentes de la interacción con la empresa y su oferta en correspondencia a los diferentes momentos de contacto o puntos de contacto (LaSalle y Britton, 2003; Shaw e Ivens, 2005). De acuerdo con 
Brakus, Schmitt y Zarantonello (2009), la experiencia del consumidor varía en intensidad, lo que significa que ciertas experiencias son más fuertes o más intensas que otras. Las experiencias varían en valencia, es decir, algunas son más positivas que otras, y algunas experiencias pueden ser negativas.

De Farias et al. (2014) señalan que, en el contexto del consumo y, en especial, de los servicios, entre los determinantes de la experiencia se encuentran las expectativas y la interacción que el individuo establece con la marca, mientras que, como consecuencia de la experiencia que el cliente obtiene, este puede llegar a experimentar cambios respecto a lo que piensa, siente y cómo actúa ante la marca (Grace y O'Cass, 2004). En relación a los determinantes, y siguiendo esta misma línea de trabajo, Schmitt y Zarantonello (2013) y, previamente, Voss y Zomerdijk (2007) señalan que la experiencia de consumo viene determinada por varios factores: por un lado, por el uso o práctica, y por otro, por el hábito o costumbre que el individuo tiene en relación a un producto o servicio.

El primero de tales factores es resultado de la relación actual del cliente con la marca y conecta de forma directa la experiencia con las percepciones, los sentimientos y las observaciones directas que resultan de la interacción entre ambos (Wei et al., 2016; Voss y Zomerdijk, 2007), por lo que sobre la misma influyen los puntos de contacto que un cliente tiene con la empresa, ya sea el entorno donde se da la relación (físico o virtual) o los individuos con los que interactúa (empleados que entregan el servicio, personal de atención al cliente, etc.). Así, a medida que un cliente usa un producto o servicio, más experiencia tiene sobre él, con la marca y, por último, con el proveedor (ya sea fabricante o distribuidor). Por otra parte, el hábito hace referencia a la costumbre que tiene el cliente de usar la marca, viniendo esto determinado por un pasado común entre ellos y por la existencia de los conocimientos y sentimientos, e incluso al nivel de lealtad que el individuo tiene desde hace tiempo (Lemon y Verhoef, 2016; Voss y Zomerdijk, 2007). Este factor viene determinado por la capacidad de la marca para cumplir las promesas que realiza al cliente, por tanto, por el grado de compromiso que esta adquiera con el cliente. Asimismo, condiciona el significado que la marca tiene para el cliente y, por tanto, las expectativas que este tiene en relación a la marca (Esch et al., 2012).
Finalmente, cabe señalar que, atendiendo al trabajo de Blackston y Lebar (2015), el resultado positivo o negativo de la experiencia viene determinado por:

- La interpretación de toda la información sensorial que recibe del exterior durante su interacción con la empresa o la marca.

- Las expectativas que el individuo tenía en relación a su vinculación con la marca.

El resultado se almacena en la memoria a largo plazo (Carbone y Haeckel, 1994), y su impacto sobre el comportamiento del cliente tiene importantes implicaciones prácticas para las marcas, tanto si actúan en entornos offline como online.

\subsection{La experiencia del cliente en el con- texto universitario online}

El concepto de «experiencia» resulta consistente con diversos enfoques actuales de marketing, tales como la lógica dominante del servicio (Vargo y Lusch, 2008) y la noción de valor de uso del producto (Tynan y McKechnie, 2009). De acuerdo con ellos, el valor se crea durante el uso y el consumo del producto, siendo el cliente quien determina el valor y quien, además, participa de forma activa en su creación (Vargo y Lusch, 2008). Las percepciones y las expectativas del cliente en relación a la marca -o empresa- son claves en la definición del valor. Así, en gran medida, el resultado final vendrá determinado por los servicios y los recursos que la organización utiliza, o el modo en que desarrolle el proceso de relación con el cliente. Pero, también, el resultado se ve influido por la capacidad

Considerar que los estudiantes son clientes de los servicios que ofrece la universidad es un tema que hoy día no genera dudas, en particular desde que el EEES ha puesto énfasis en la evaluación del valor y en las cuestiones relativas a la percepción y a la experiencia del estudiante en las aulas 


\section{El entorno virtual de aprendizaje adquiere una importancia fundamental en la experiencia obtenida por el estudiante, ya que la actividad de mediación que desarrolla la tecnología en este caso condiciona el tipo de experiencia que obtiene el alumno}

de la organización -o la marca- para cumplir con las expectativas y los objetivos del cliente (Lemke, Clark y Wilson, 2011).

Abundantes trabajos han analizado el concepto "valor de uso del cliente» en una amplia gama de servicios en los que la presión competitiva resulta elevada, como el turismo (Morgan, Lugosi y Brent, 2010), los servicios financieros (Sharma y Patterson, 2000) 0 la distribución online (Rose et al., 2012). Ahora bien, dado que recientemente la presión competitiva ha crecido hasta alcanzar sectores como el de la asistencia sanitaria (Propper, Wilson y Burgess, 2006), los servicios públicos y la educación (Goldhaber y Eide, 2003), especialmente en países que han logrado elevadas cotas del estado del bienestar, cada vez resulta más necesario analizar el mencionado concepto en este otro tipo de servicios. En particular, la universidad española no es ajena a una realidad donde la caída de la inversión pública está obligando a las instituciones académicas a desarrollar modelos de gestión basados en la autofinanciación. Junto a esta razón, también se ha de considerar el incremento de las tasas académicas ( $\mathrm{Ng}$ y Forbes, 2009) y la posibilidad cada vez mayor de que los estudiantes encuentren oportunidades de aprendizaje/investigación en todo el mundo. Ante el incremento de la competencia entre instituciones universitarias (Munteanu et al., 2010), no es de extrañar que la experiencia del estudiante comience a ser una variable relevante en los modelos de gestión universitaria (Asthana, 2006).

Considerar que los estudiantes son clientes de los servicios que ofrece la universidad es un tema que hoy día no genera dudas, en particular desde que el Espacio Europeo de Educación Superior (EEES) ha puesto énfasis en la evaluación del valor y en las cuestiones relativas a la percepción y a la experiencia del estudiante en las aulas. Como ya se señaló con anterioridad, una inmersión en un contexto experiencial conlleva el consumo de servicios (Cova y Dalli, 2009), y la educación superior es un servicio de alta complejidad, especialmente cuando se ofrece en el entorno online, donde el nivel de interactividad y cambio es tan elevado que fomentan la creación de experiencias ( $\mathrm{Ng}$ y Forbes, 2009).

La experiencia de los estudiantes se puede entender como el resultado de la totalidad de la interacción del estudiante con la institución. Siguiendo la propuesta de Schmitt y Zarantonello (2013) anteriormente presentada, es posible señalar que la experiencia del estudiante viene determinada por el conocimiento y la experiencia que el individuo tiene sobre la institución, así como por el contexto donde la relación tiene lugar.

La interacción estudiante-institución va cambiando a medida que transcurre el tiempo. Así, mientras que en las primeras fases de la relación, los miembros no se conocen, a medida que el tiempo pasa, la relación se convierte en habitual y el conocimiento se incrementa. En este punto, la relación se basa en la confianza y en el compromiso, dado que se ha demostrado la capacidad de los miembros para cumplir las promesas realizadas (Chang et al., 2014). De este modo, una relación satisfactoria entre el estudiante y la institución constituye una de las bases para el desarrollo de una experiencia de cliente óptima (Bishop, 2015). Por lo que respecta al contexto, en la universidad online, todas las relaciones que se producen entre los estudiantes, el personal académico y de gestión de la institución y los recursos docentes se llevan a cabo a través de un entorno virtual de aprendizaje. Este adquiere una importancia fundamental en la experiencia obtenida por el estudiante, ya que la actividad de mediación que desarrolla la tecnología en este caso condiciona el tipo de experiencia que obtiene el alumno (Cabero y Llorente, 2009).

\subsection{Los determinantes de la experiencia del estudiante universitario online}

Un aspecto que caracteriza a los servicios es que los consumidores participan de forma directa en los procesos de producción y entrega. En muchos servicios, se 
requiere que el consumidor aporte información o esfuerzo antes de que la transacción de servicio tenga lugar (Kelley, Donnelly y Skinner, 1990). El input del consumidor constituye la materia prima transformada por los empleados de la organización en un servicio. En consecuencia, el consumidor contribuye directamente a la calidad del servicio prestado y a su propia (in)satisfacción (Lemke, Clark y Wilson, 2011).

Si los inputs proporcionados por el consumidor son inadecuados 0 inapropiados, esto puede conducir a problemas o deficiencias del servicio.

En el ámbito de la educación superior, los ejemplos podrian incluir a los estudiantes que no han hecho la preparación previa necesaria para los tutoriales y para realizar preguntas apropiadas en los tutoriales, el omitir la entrega del trabajo para su evaluación, no prestar suficiente atención durante las clases, etc. Por lo tanto, la productividad y la calidad del servicio no solo dependen de la actuación del personal del proveedor de servicios, sino también del comportamiento del consumidor, que, a su vez, puede hacer que la gestión de la calidad sea problemática.

Sin embargo, se debe señalar que el input suministrado por el estudiante también viene determinado por el entorno donde este desarrolla el proceso de aprendizaje, así como también por el tipo de relación que el mismo establece con el suministrador del servicio. Las características del entorno donde se desarrolla el proceso de aprendizaje condicionarán el servicio recibido. Así, la naturaleza presencial o virtual del mismo, la disponibilidad de determinadas herramientas de comunicación o el acceso a un elevado número de recursos de aprendizaje pueden incrementar la calidad del servicio recibido.

Por otro lado, se ha de señalar que muchos servicios requieren una estrecha interacción personal entre un proveedor de servicios y el consumidor. El hecho de que el consumidor considere o no que tal interacción es satisfactoria puede depender de una variedad de factores que van desde la apariencia del proveedor de servicios (ya sea el personal académico o de apoyo) y su percibida competencia, hasta las características de la personalidad y la atracción interpersonal entre los participantes. Aunque algunos de estos factores pueden contribuir a la heterogeneidad y a la variabilidad del servicio, no son fáciles de controlar.

La figura 1 presenta el modelo conceptual para la experiencia del estudiante universitario online propuesta. Considerando las ideas anteriores, este modelo propone que la experiencia del estudiante viene determinada por la calidad del entorno virtual de aprendizaje y por la valoración realizada sobre la marca de la universidad virtual.

\section{Figura 1. Modelo conceptual}

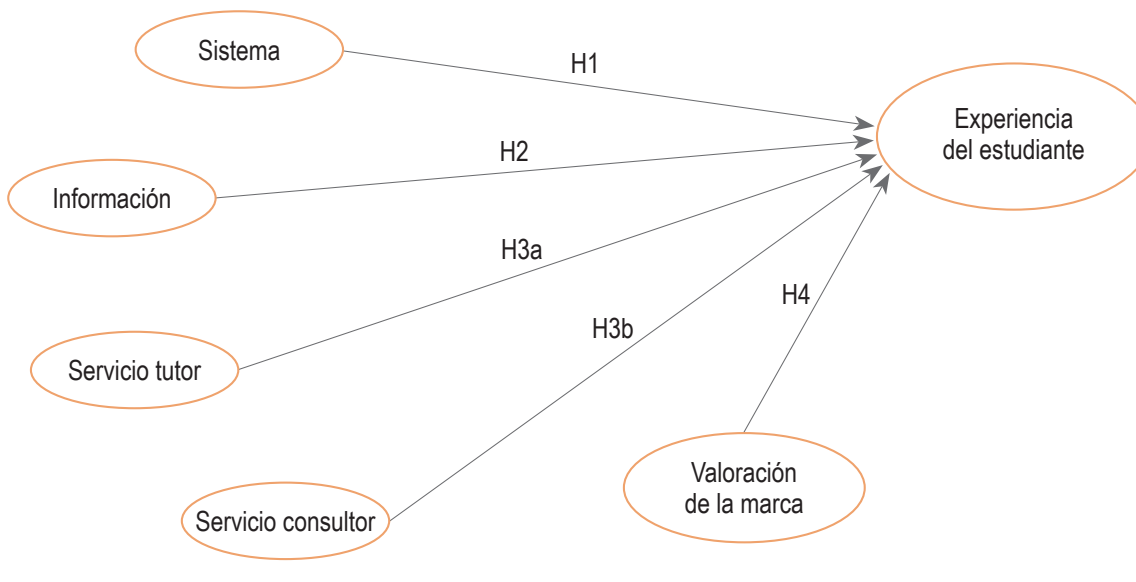

Fuente: elaboración propia 


\subsubsection{La calidad del aula virtual}

En los entornos B2C, la calidad del sitio web es señalada como un factor clave en el comercio electrónico, ya que la percepción del cliente en relación a la calidad del sitio genera un impacto positivo en su intención de uso (Chang y Chen, 2008), a la vez que afecta directamente a las intenciones de compra (Lin, 2007). En esta misma línea, Shapiro et al. (2017) señalan que la experiencia del cliente online viene condicionada por la calidad de la web, ya que la misma condiciona en gran medida el tipo de relación que establece con la marca y el resultado que obtiene de la misma.

Diversos trabajos han establecido metodologías para medir la calidad del sitio web, identificando en ellos las diferentes dimensiones que conforman este constructo. Uno de los modelos que mayor difusión ha alcanzado es el propuesto por Lin (2007). Según este modelo, la calidad de un sitio web es multidimensional y comprende la calidad del sistema, la calidad de la información y la calidad del servicio. Uno de los principales atractivos del modelo es que explica la calidad adoptando un doble enfoque basado en (Hartono et al., 2014):

- Sistema de información.

- Cliente.

Pansari y Kumar (2017) señalan que, bajo un enfoque centrado en el sistema de información, los determinantes de la aceptación del cliente se basan en características, tales como sistemas de usabilidad web, información precisa y seguridad de las transacciones, mientras que una orientación basada en el cliente muestra que la atracción y la retención del cliente en el servicio online vienen determinadas por las características de la

\section{[...] la calidad del sitio web es} señalada como un factor clave en el comercio electrónico, ya que la percepción del cliente en relación a la calidad del sitio genera un impacto positivo en su intención de uso relación establecida con el vendedor durante el servicio online. En particular, Lin (2007) señala que características tales como la capacidad de respuesta, la confianza o la empatía mostrada por el vendedor son determinantes a la hora de establecer la calidad del trato dado al cliente. Ambos puntos de vista de la investigación son aplicables en los servicios de educación superior. Por lo tanto, las características técnicas del aula en línea se identifican con el sistema, mientras que los materiales docentes se corresponderán con la información. Finalmente, la relación que se establezca entre el profesorado y los estudiantes determinará el servicio.

Con respecto a la primera de las dimensiones señaladas, la calidad del sistema, cabe señalar que hace referencia a la tecnología que hace posible el funcionamiento del aula dentro del entorno virtual de aprendizaje. Elementos como la facilidad de uso, el diseño web o la conveniencia determinan la calidad percibida por el estudiante (Bharati y Chaudhury, 2004). De esta forma, encontramos que los niveles de interactividad influyen significativamente sobre el nivel de uso que el estudiante realiza de la información que suministra la web (Palmer, 2002). Asimismo, la interfaz determina parte del atractivo percibido por el estudiante en el entorno de aprendizaje, condicionando el nivel de interacción del estudiante con el resto de miembros. Por otra parte, los estudiantes necesitan de una retroalimentación o respuesta por parte del profesorado y del personal de la institución académica. Finalmente, es posible apuntar al respecto que los trabajos de Kauffman (2015) y de Wu, Shen y Chang (2015) señalan que el diseño web y la interactividad entre las partes constituyen elementos fundamentales en el éxito del proceso de aprendizaje. En particular, los trabajos apuntados consideran que estos dos factores inciden en el nivel de satisfacción del estudiante y en su predisposición a realizar procesos de aprendizaje colaborativo.

La segunda de las dimensiones mencionadas, la calidad de la información, se mide en términos del valor que recibe el estudiante de los contenidos informativos y de los recursos didácticos que se encuentran en el aula virtual. En relación a los mismos, trabajos como los de DeLone y McLean (2003) enfatizan que características relativas a la actualización de contenidos, a la utilidad, a la precisión y a la presentación de la información son fundamentales a la hora de de- 
terminar la calidad. Por otro lado, algunos trabajos en el ámbito del e-commerce señalan que, en relación a la calidad de la información, también se han de considerar los aspectos relativos a la seguridad y a la confidencialidad de la información, en especial aquella relativa a los datos personales y financieros del cliente (Vijayasarathy, 2004; Janda et al., 2002). En este sentido, en un entorno virtual de aprendizaje, la confidencialidad de este tipo de información y la referida a datos de matriculación -como calificaciones, etc.- también resulta fundamental (D'Este, Castro y Molas-Gallart, 2009). En concreto, Xiao y Wilkins (2015) sugieren que la calidad de la información ayuda a mejorar la relación entre el estudiante y la institución, reduciendo los niveles de incertidumbre y mejorando sus niveles de satisfacción.

Finalmente, la tercera de las dimensiones apuntadas previamente, la calidad del servicio, se define como la evaluación y el juicio que el estudiante tiene en relación al proceso de enseñanza recibido (Chong y Ahmed, 2015). La calidad del servicio se reconoce como un elemento determinante en la intención del estudiante de matricularse en la universidad online. Trabajos como los de DeLone y McLean (2003) pusieron de manifiesto que la capacidad de respuesta, la confianza y la empatía son factores críticos para que la percepción de la calidad del servicio en el entorno online sea elevada. Ya a comienzos de los años ochenta del siglo pasado, los trabajos de Parasuraman, Zeithaml y Berry (1985 y 1988) apuntaban a la relevancia de estos factores sobre la percepción de la calidad del servicio en entornos presenciales. Posteriormente, el trabajo de Akbar y Mannan (2015) observó que estos factores eran clave en servicios online, como la educación.

En relación a la educación online, es preciso apuntar que el servicio recibido por el estudiante es ofrecido por el docente, figura en la que cabe realizar la distinción posterior entre profesor-tutor y profesor-consultor. De este modo, la figura del profesor-consultor hace referencia al docente que está encargado del aprendizaje del estudiante en una determinada materia, así como de evaluar de forma continua su progreso académico y de ofrecer un feedback, mientras que el profesor-tutor se ocupa del diseño curricular del estudiante, así como también de actuar como mediador entre el estudiante y la institución ante cualquier proceso o, incluso, ante cualquier problema -por el que el estudiante ha de pasar-. Ambos perfiles se consideran fundamentales.

\section{Según Lin (2007), la calidad de un sitio web es multidimensional y comprende la calidad del sistema, la calidad de la información y la calidad del servicio}

Atendiendo a las ideas expuestas previamente, es posible formular las siguientes hipótesis de investigación:

- H1. La calidad percibida en el sistema influye en la experiencia del estudiante en relación a la universidad.

- H2. La calidad percibida en la información influye en la experiencia del estudiante en relación a la universidad.

- H3. La calidad percibida en la relación entre el estudiante y el docente influye en la experiencia del estudiante en relación a la universidad.

Ahora bien, teniendo en cuenta la distinción previamente señalada entre la figura del profesor-tutor y la del profesor-consultor, la hipótesis H3 puede subdividirse en las siguientes dos subhipótesis, que distinguen entre que la relación del estudiante se refiera a la relación con el docente-tutor $(\mathrm{H} 3 \mathrm{a}) \mathrm{o}$ a la relación con el docente-consultor $(\mathrm{H} 3 \mathrm{~b})$ :

- H3a. La calidad percibida en la relación entre el estudiante y el profesor-tutor influye en la experiencia del estudiante en relación a la universidad.

- H3b. La calidad percibida en la relación entre el estudiante y el profesor-consultor influye en la experiencia del estudiante en relación a la universidad.

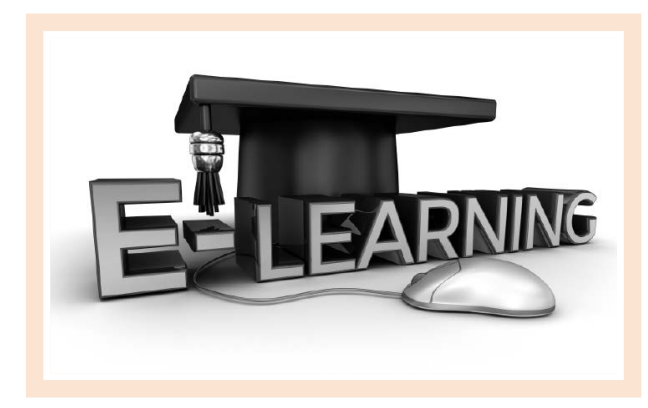




\section{[...] la marca proporciona una señal} o una promesa a los consumidores sobre el producto o servicio que se ofrecerá, mitigando así algunos de los problemas asociados con la experiencia y las cualidades credenciales

\subsubsection{La valoración sobre la marca de la uni- versidad}

La marca de la institución académica ejerce una gran influencia sobre el comportamiento de los estudiantes. Este nombre, término o símbolo ofrece protección, pero, sobre todo, permite identificar y diferenciar a la universidad del resto de instituciones académicas. Aaker (1991) describe la marca como un logo o nombre que sirve para diferenciar los productos o servicios de los diferentes suministradores/fabricantes. Sin embargo, Marconi (1993) señala que la marca no es solo el nombre, puesto que el nombre es creado para identificar el producto, mientras que la marca es creada para añadir valor al producto y darle personalidad.

En esencia, la marca proporciona una señal o una promesa a los consumidores sobre el producto o servicio que se ofrecerá, mitigando así algunos de los problemas asociados con la experiencia y las cualidades credenciales (De Chernatony y McDonald, 1998). En esta situación, la marca puede jugar un importante papel como mitigadora del riesgo, dando a los consumidores una mayor confianza en su proceso de toma de decisiones e incrementando la confianza (Erdem y Swait, 1998). Además de como mitigadora del riesgo, ya que la marca es una fuente de información, también puede servir como una herramienta para diferenciar y facilitar el proceso de elección de los consumidores mediante la creación de un carácter distintivo. Así, la marca se ha reconocido cada vez más como un determinante importante de la elección del consumidor en el sector de los servicios (Turley y Moore, 1995).

En este sentido, Balmer y Liao (2007) sugieren que la marca universidad ofrece a los graduados un sentido de identificación y una forma de definirse a sí mismos no solo como clientes, sino también como miembros de toda una organización de marca corporativa. A este respecto, ya que la universidad es una entidad que presta un servicio, Lowrie (2007) explica que la marca universidad debe prestar atención a los aspectos de intangibilidad e inseparabilidad de los servicios educativos. Por lo tanto, como una marca de servicio, requiere un mayor énfasis en el marketing interno, en parte, ya que todos los empleados se convierten en puntos de contacto del consumidor. Las marcas son esenciales para el estatus social de los consumidores, y, de hecho, un aspecto de la universidad es el otorgamiento de un cierto nivel de estatus social (Williams y Omar, 2014).

Por medio de las connotaciones asociadas a la marca, esta hace posible que el estudiante obtenga un primer conocimiento sobre la universidad, facilitando así la toma de decisiones relativas a cursar sus estudios en ella. En este sentido, Pimienta et al. (2016) señalan que la imagen que el estudiante tiene sobre la institución universitaria se forma a lo largo del tiempo, de tal manera que a ella se vincula todo un sistema de valores y asociaciones que se mantienen de manera más o menos estable a lo largo del tiempo. Pero, además, la marca aporta información relevante sobre el comportamiento de la institución académica y, sobre todo, de su capacidad para cumplir sus promesas (Dennis et al., 2017). Esta información es de vital importancia para el estudiante a la hora de decidir su permanencia en la institución, consolidando con ello su relación en el tiempo.

Chang y Chen (2008) sugieren que el conocimiento y la imagen de marca constituyen elementos determinantes en el proceso de valoración de la marca. Ambos no solo favorecen la toma de decisiones en situaciones con diferente nivel de incertidumbre, sino que además hacen posible el desarrollo de una relación entre el estudiante y la institución basada en la confianza y en el compromiso mutuo (Balaji, Roy y Sadeque, 2016).

En esta línea, los trabajos de Patlan-Pérez, Martínez y Hernández (2012) y de Polat (2011) señalan que, en las instituciones universitarias, la imagen de marca es un factor importante para atraer y retener a los mejores estudiantes, profesores y empleados. En particular, estos autores señalan que, para los estudiantes, la imagen de una institución educativa es fundamental para elegir la universidad, fomentar su lealtad y atraer a otros estudiantes. Mientras que, en lo referente a los profesores y al personal de apoyo, la imagen de la institución resulta 
primordial en el incremento de su desempeño y productividad, a la vez que genera vínculos afectivos, mayor compromiso y cohesión entre el personal, y entre este y la institución (Patlan-Pérez y Martínez-Torres, 2017; Traverso-Cortes, 2005).

En concreto, la valoración que se realiza sobre la marca influye de forma directa sobre la experiencia del estudiante con respecto a la institución (Hsieh y Chang, 2016). Las expectativas que el estudiante deposita en la institución académica se refieren a la obtención de un resultado académico determinado, pero, también, tienen que ver con los procesos y los comportamientos

\section{METODOLOGÍA}

El trabajo de campo de esta investigación se llevó a cabo mediante el envío de un correo electrónico con el enlace de acceso a la encuesta a los alumnos matriculados en los diferentes programas de posgrado de una universidad especializada en enseñanza virtual, la UOC ${ }^{5}$. El número de alumnos matriculados en el curso 2012/2013 fue de 2.003 alumnos, de los cuales respondieron el cuestionario un total de 304 . Los datos aparecen reflejados en la ficha técnica (véase tabla 1). Los datos han sido obtenidos a partir de una que esta desarrolla durante la relación que establece con el estudiante. Si el estudiante percibe que la institución se implica en la relación y cumple de forma satisfactoria los compromisos adquiridos, es muy posible que este vea cumplidas sus expectativas en grado sumo, de forma que la experiencia obtenida sea muy favorable.

En función de las ideas previas es posible plantear la siguiente hipótesis de investigación:

- H4. La valoración realizada sobre la marca de la institución influye de forma positiva en la experiencia del estudiante en relación a la universidad. encuesta que cada semestre realiza la propia universidad a todos sus estudiantes.

El cuestionario está dividido en seis secciones, en las cuales se pregunta sobre diferentes aspectos relacionados con las aplicaciones tecnológicas y los contenidos informativos, así como por la actividad desarrollada por los profesores-tutores y consultores. Asimismo, se incluye una sección en la cual se pregunta a los estudiantes por la valoración que realizan de la marca institucional.

\section{Tabla 1. Ficha técnica}

\begin{tabular}{|c|c|}
\hline Universo ....... & $\begin{array}{l}\text { Estudiantes de los programas de posgrado en el segundo semestre del curso académico } \\
2012 / 2013 \text { de la UOC. }\end{array}$ \\
\hline 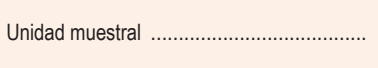 & $\begin{array}{l}\text { Estudiantes de los programas de posgrado en el segundo semestre del curso académico } \\
2012 / 2013 \text { de la UOC. }\end{array}$ \\
\hline Método de recogida de información ............. & E-mail enviado a los alumnos, adjuntando un enlace para rellenarlo. \\
\hline 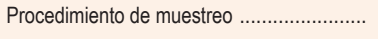 & Método no probabilístico, de conveniencia. \\
\hline 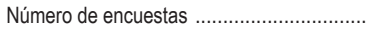 & 2.003 alumnos (304 encuestas recibidas). Tasa de respuesta del $15 \%$. \\
\hline Periodo de recogida de la información ........ & Del 18 de julio de 2014 al 10 de septiembre de 2017. \\
\hline
\end{tabular}

Fuente: elaboración propia

\footnotetext{
5 Para más información, véase www.uoc.edu.
} 


\subsection{Las variables del estudio}

Las variables utilizadas en el análisis de datos se muestran en la tabla del apéndice 1. Las variables empleadas permiten medir la calidad percibida del aula virtual y la valoración de la marca que realizan los estudiantes en relación a dicha universidad. Para medir la calidad del aula virtual se ha utilizado la propuesta de Lin (2007), en la que:

- La calidad del sistema viene determinado por el diseño de la web y el grado de interactividad.

- La calidad de la información está determinada por la capacidad informativa y la seguridad.

- La calidad del servicio viene determinada por la capacidad de respuesta, la confianza y la empatía.

En relación con la calidad del sistema, el diseño web hace referencia al nivel de usabilidad, acceso, conveniencia, facilidad de uso y atractivo que la plataforma web presenta, mientras que la interactividad está relacionada con la capacidad de la que dispone el usuario de la web, en este caso el estudiante, de participar de forma interactiva en el proceso gracias a la presencia de herramientas multimedia. La interactividad incluye los mecanismos de feedback y respuesta múltiple.

Por lo que respecta a la calidad de la información, la primera dimensión valorada es la capacidad informa-

\section{ANÁLISIS DE RESULTADOS}

A diferencia de otras investigaciones, donde se puede obtener un perfil psicográfico de la muestra, en este caso, no es posible. Este hecho viene motivado por tratarse de encuestas de calidad de la docencia, por lo tanto, no se han podido obtener aquellos datos relacionados con aspectos económicos del alumno, composición del hogar, estilos de vida, etc.

El primer paso fue realizar un análisis factorial exploratorio con el fin de analizar la unidimensionalidad de las escalas. En dicho análisis se realizó la prueba de esfericidad de Bartlett, que, mostrando un p-valor de 0,000 , permitió rechazar la hipótesis nula de variables incorrelacionadas, siendo, por tanto, idóneo aplicar el análisis factorial. Además, el valor del KMO (KaiserMeyer-Olkin) obtenido está cercano a la unidad. tiva, la cual hace referencia a la habilidad de informar al usuario, de forma precisa y eficiente, sobre los servicios o productos disponibles en la web. Cuando hacemos referencia a recursos o contenidos docentes, la capacidad informativa se refiere a la capacidad de los mismos para facilitar el aprendizaje o mejorar el grado de formación del estudiante, dados la precisión, el grado de novedad o la utilidad de los materiales docentes. La segunda dimensión considerada es la seguridad, la cual hace referencia a la confidencialidad de la información personal y académica del estudiante.

En relación a la calidad del servicio, las dimensiones consideradas son la capacidad de respuesta, la confianza y la empatía, tanto de los profesores como de los tutores. La capacidad de respuesta hace referencia al nivel de utilidad que el estudiante percibe en la respuesta dada por los profesores-consultores y los tutores. Mientras que la confianza indica la percepción de credibilidad que para el estudiante tiene el profesor-consultor y el profesor-tutor. Por último, la empatía hace referencia a la capacidad del profesorado, tutor y consultor, para comprender y dar una respuesta adecuada a las necesidades del estudiante. Finalmente, se pide al alumno que valore la marca «universidad» desde su experiencia como alumno.

A continuación, se procedió a comprobar el grado de fiabilidad de las diferentes escalas utilizadas, calculando para ello el alfa de Cronbach para cada dimensión y para cada grupo. El valor obtenido en todos los constructos superó el 0,7 recomendado por Nunnally (1978).

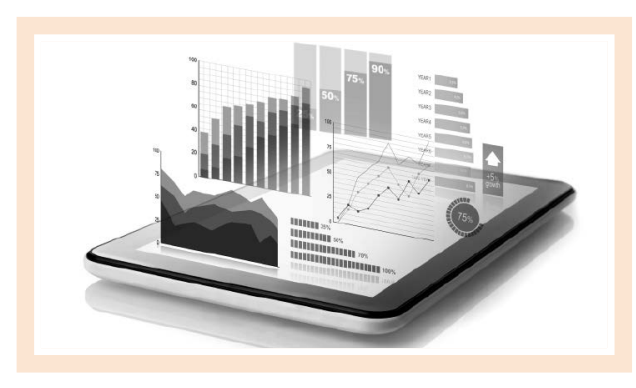


La depuración definitiva de las escalas (véase tabla del apéndice 2) se basó en la metodología de desarrollo de modelos estructurales (Hair et al., 1999). Esta técnica consiste en eliminar de forma progresiva y de uno en uno aquellos ítems que incumplan alguno de los tres criterios propuestos por Jöreskog y Sörbom ${ }^{6}$ (1993).

En una primera etapa se planteó la inexistencia de dimensiones para cada constructo y, por tanto, la totalidad de los ítems cargaba sobre un único factor. Una vez realizados los análisis factoriales de primer orden para cada una de las variables latentes, se detectó:

- La existencia de correlaciones entre los errores de los indicadores que componían cada constructo.

- Algunos de los valores lambda $(\lambda)$ eran inferiores a 0,5 .

Por ello, antes de proceder a la eliminación de dichos ítems se practicó a cada constructo el LM-test y el test de Wald para comprobar la posible existencia de otra estructura subyacente o para detectar posibles modificaciones que mejoraran los resultados obtenidos.

Dicho análisis puso de manifiesto que cada constructo estaba formado por diferentes dimensiones, lo que condujo a la realización de una estrategia de modelos rivales (Hair et al., 1999). Uno de los modelos rivales considerado fue el analizado anteriormente, es decir, un modelo sin dimensiones, siendo el modelo alternativo rival aquel en el que se reflejan las diferentes dimensiones analizadas en la literatura.

${ }^{6}$ En concreto, los tres criterios propuestos son:

a) Convergencia débil (Steenkamp y Van Trijp, 1991), que implica eliminar los indicadores que no presenten coeficientes de regresión factorial significativos ( $t$ student $>2,58 ; p=0,001$ ).

b) Convergencia fuerte (Steenkamp y Van Trijp, 1991), que supone eliminar los indicadores no sustanciales, es decir, aquellos coeficientes estandarizados que sean menores a 0,5 .

c) Jöreskog y Sörbom (1993) proponen eliminar los indicadores que menos aporten a la explicación del modelo $\left(R^{2}<0,3\right)$.

Debemos destacar que estos criterios fueron aplicados en el orden en el que han sido planteados y que la eliminación de ítems se fue desarrollando de manera individual, de tal forma que tras la eliminación de un ítem se generaba un nuevo modelo factorial que debía ser evaluado.
Finalmente, se comprobó que el modelo que contenía las diferentes dimensiones de cada constructo ajustaba mejor que el modelo de partida.

Por último, y de forma complementaria a los análisis de fiabilidad llevados a cabo en la etapa exploratoria, se utilizaron dos criterios adicionales para medir la consistencia de las escalas propuestas: la fiabilidad compuesta del constructo (FCC) y el análisis de la varianza (AVE). Como se puede apreciar, en todos los constructos se han alcanzado valores considerados como adecuados.

El siguiente paso fue validar el modelo estructural recogido en la figura 1 mediante la técnica de regresión por mínimos cuadrados parciales (PLS). El modelo se estimó mediante SmartPLS 2.0 (Ringle, Wende y Will, 2005). La significatividad de los parámetros se estableció mediante un proceso de remuestreo por Bootstrap de 304 submuestras de tamaño igual a la muestra original.

Para garantizar la validez convergente fueron suprimidos todos aquellos indicadores cuya carga factorial era no significativa o inferior a 0,7 . El modelo resultante no evidencia problemas de fiabilidad atendiendo a cualquiera de los criterios establecidos (alfa de Cronbach, fiabilidad compuesta, varianza extraída promedio) (véase tabla 2).

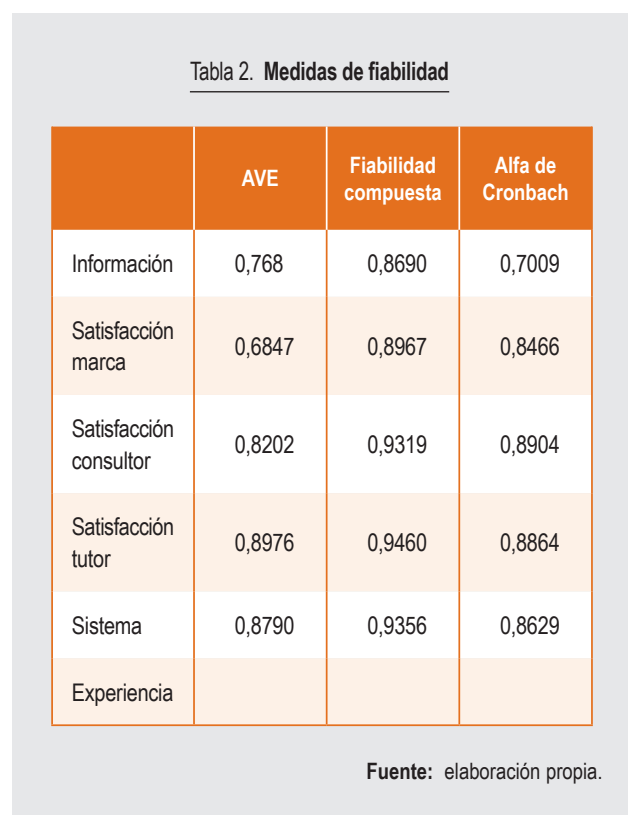


Para evaluar la validez discriminante, se ha recurrido al único criterio que es aplicable en la estimación mediante PLS, que es el que indica que la varianza promedio extraída para cada factor debe ser superior al cuadrado de la correlación entre cada par de factores (Fornell y Larcker, 1981), como así queda reflejado en la tabla 3.
Para valorar la capacidad predictiva del modelo estructural se ha seguido el criterio planteado por Falk y Miller (1992), según el cual el $R^{2}$ de cada constructo dependiente debe ser superior al valor 0,1 . En la tabla 4 aparecen los valores correspondientes, así como el resultado del contraste del modelo planteado en la figura 2.

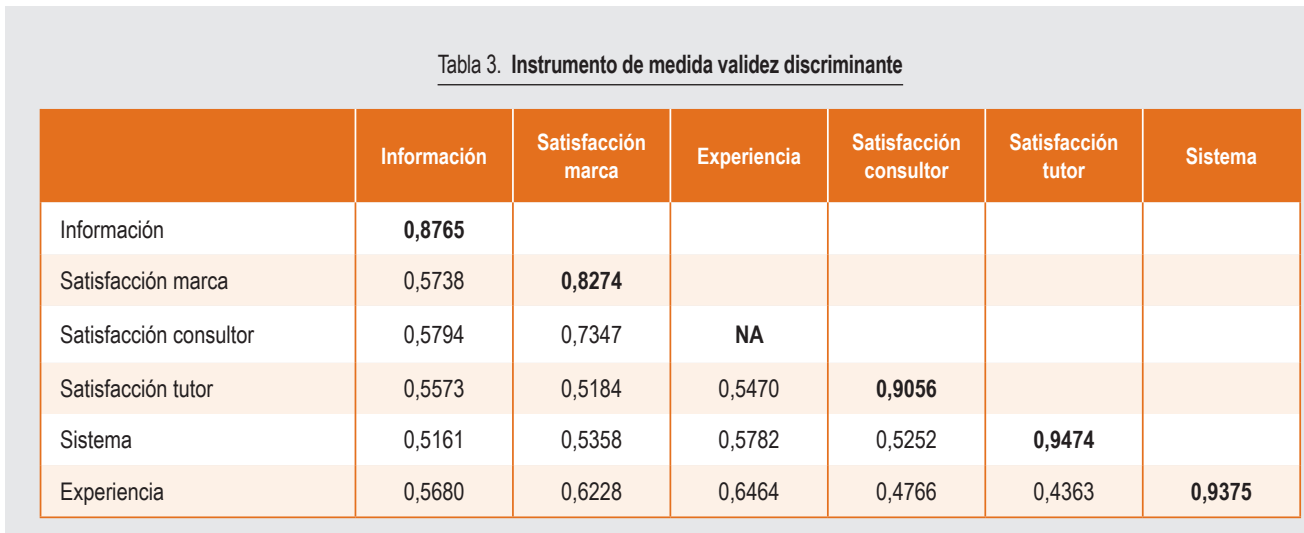

Debajo de la diagonal: correlación estimada entre los factores.

Diagonal: raíz cuadrada de la varianza extraída.

$\mathrm{NA}=$ no aplicable

Fuente: elaboración propia.

\section{Tabla 4. Contraste de hipótesis}

\begin{tabular}{|c|c|c|}
\hline Hipótesis & $\begin{array}{c}\beta \\
\text { estandarizado }\end{array}$ & $\begin{array}{l}\text { Valor t } \\
\text { Bootstrap }\end{array}$ \\
\hline $\begin{array}{l}\text { H1. La calidad percibida en el sistema influye en la experiencia del estudiante en relación } \\
\text { a la universidad. }\end{array}$ & $0,231^{\star \star *}$ & 3,0372 \\
\hline $\begin{array}{l}\text { H2. La calidad percibida en la información influye en la experiencia del estudiante en relación } \\
\text { a la universidad. }\end{array}$ & $0,073^{\mathrm{NS}}$ & 1,3766 \\
\hline $\begin{array}{l}\text { H3a. La calidad percibida en la relación entre el estudiante y el profesor-tutor influye en la } \\
\text { experiencia del estudiante en relación a la universidad. }\end{array}$ & $0,095^{\star *}$ & 2,1679 \\
\hline $\begin{array}{l}\text { H3b. La calidad percibida en la relación entre el estudiante y el profesor-consultor influye en } \\
\text { la experiencia del estudiante en relación a la universidad. }\end{array}$ & $0,172^{\star \star *}$ & 3,9095 \\
\hline $\begin{array}{l}\text { H4. La valoración realizada sobre la marca de la institución influye de forma positiva en la } \\
\text { experiencia del estudiante en relación a la universidad. }\end{array}$ & $0,408^{\star * *}$ & 6,2755 \\
\hline
\end{tabular}

$$
\begin{aligned}
& R^{2} 0,642 \\
& { }^{*} p<0,10 \\
& { }^{* *} p<0,05 \\
& { }^{* *} p<0,01 \\
& N S=\text { no significativo }
\end{aligned}
$$




\section{Figura 2. Modelo contrastado}

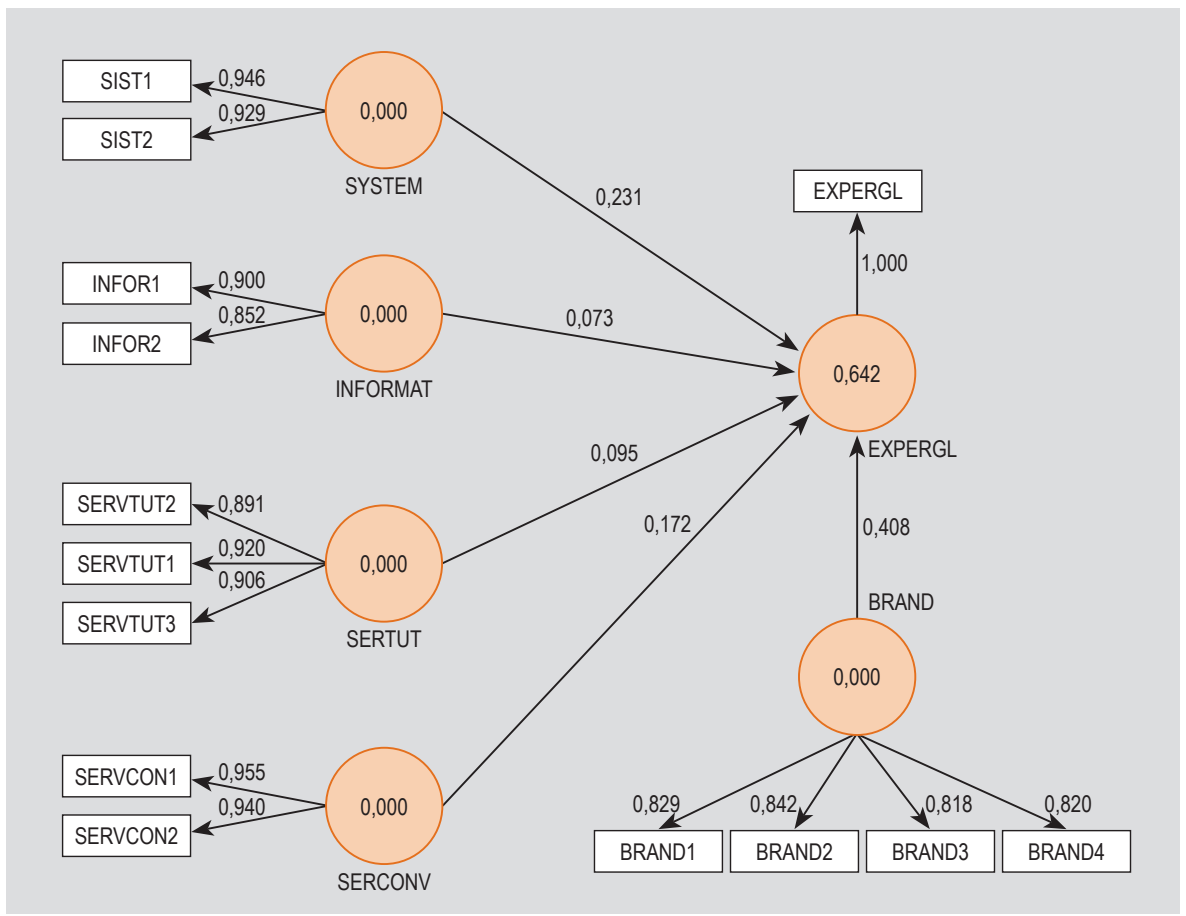

Los resultados obtenidos confirman, por un lado, que los efectos directos y más intensos sobre la experiencia provienen de la importancia que tiene la marca universidad ( $\beta=0,408 ; p<0,01 ; H 4)$. Este resultado es coherente con el planteamiento de la importancia de la marca universidad para disminuir la incertidumbre, dado el carácter intangible del servicio que recibe el estudiante. Así, de acuerdo con los resultados mostrados por Patlan-Pérez y Martínez-Torres (2017), Pollat (2011), o previamente por Chang y Chen (2008) y Lowrie (2007), la marca es usada como mecanismo utilizado por los estudiantes para cubrir sus necesidades de autorrealización o desarrollo profesional al adquirir dicho servicio (Balmer y Liao, 2007).

En segundo lugar, la calidad en el sistema $(\beta=0,231$; $p<0,01 ; H 1)$. Estos resultados son acordes con investigaciones anteriores, donde se pone de manifies- to la importancia que tienen aspectos relacionados con la retroalimentación, la interfaz, la navegabilidad, la facilidad de uso, etc. (Bharati y Chaudhury, 2004; Kauffman, 2015; Lin, 2007; Wu, Shen y Chang, 2015). Estos aspectos relacionados con el entorno donde se lleva a cabo el proceso de aprendizaje son clave en la valoración que el estudiante realiza. De los mismos depende una parte importante del esfuerzo y el tiempo que el individuo dedica al proceso de aprendizaje, de tal manera que el mismo puede resultar sencillo, divertido y placentero, generándose así asociaciones positivas que influyen de manera directa en una experiencia positiva.

En tercer lugar, el efecto del servicio prestado por el profesor-consultor $(\beta=0,172 ; p<0,01 ; \mathrm{H} 3 \mathrm{~b})$ presenta un peso importante sobre la experiencia del estudiante. Cuando el estudiante adquiere el servicio «enseñanza» 
lo que desea es satisfacer sus necesidades de aprendizaje, y estas necesidades solo son cubiertas por las personas que de forma directa se encargan del proceso de enseñanza-aprendizaje. Adicionalmente, esta variable también contribuye a largo plazo en la formación de la marca universidad (Balaji, Roy y Sadeque, 2016), ya que poseer de unos excelentes recursos humanos (profesores) es una señal de calidad que las universidades envían a la sociedad. Finalmente, en cuarto lugar se sitúa la calidad del servicio prestado por el profesor-tutor $(\beta=0,095 ; p<0,01 ; H 3 a)$. Aunque con un efecto más débil que los anteriores indicadores, pone de manifiesto que los estudiantes necesitan de un profesional que, ligado a la institución, les ayude en la definición de su itinerario curricular, a la vez que los oriente y los ayude

\section{CONCLUSIONES}

El presente trabajo pone de manifiesto que los modelos estratégicos de marketing que son desarrollados en sectores altamente competitivos resultan de gran utilidad para gestionar organizaciones con una finalidad más social. Así, conceptos como el de «experiencia de cliente», que actualmente están ganando gran relevancia en el diseño estratégico de marketing, tanto en entornos convencionales como online, resultan de gran utilidad en la gestión de instituciones académicas de educación superior, las cuales se ven cada vez más obligadas a seguir una orientación hacia el estudiante -cliente de sus servicios- con el fin de garantizar su supervivencia y posición en el mercado.

Siguiendo la propuesta de Schmitt and Zarantonello (2013), este trabajo propone que la calidad del aula virtual, medida a través de sus tres dimensiones (calidad del sistema, calidad de la información y calidad del servicio), junto a la valoración que el individuo hace de la marca de la universidad, son factores clave en la formación de la experiencia del estudiante como cliente. El análisis de una muestra de 306 estudiantes de posgrado de la UOC confirma tres de las cuatro hipótesis planteadas en el trabajo. De esta forma se observa que la calidad del sistema, la calidad del servicio y la valoración de la marca son factores determinantes en la experiencia del estudiante con la institución.

De forma particular, la valoración que el estudiante realiza de la marca de la universidad es el factor que a resolver consultas, problemas o cualquier otro tipo de situaciones relacionadas con trámites administrativos y académicos (DeLone y McLean, 2003). Por último, no se corrobora el efecto de la información facilitada al alumno ( $\beta=0,073 ; p<0,01 ; H 2)$, en contra de lo formulado por DeLone y McLean (2003), que destacaban la importancia de poner a disposición del alumno todo tipo de información actualizada, ordenada e importante. Esto puede venir motivado por la estandarización de la información y el uso cada vez mayor de recursos en la plataforma facilitada por las universidades para su fácil y rápida localización por parte de los estudiantes (utilización del mismo tipo de plataformas de enseñanza, diseños de web parecidos), así como la transparencia que de la información intentan transmitir las universidades.

mayor peso presenta sobre la experiencia. Este hecho se evidencia si atendemos a las elevadas tasas de fidelización que muestra la comunidad de estudiantes y graduados de la universidad -superior al $70 \%-$, asi como a la fuerte imagen que esta tiene asociada como una institución líder en la formación online. Cabe señalar que esta universidad es la primera en ofrecer enseñanza $100 \%$ online, de tal modo que ha conseguido consolidar una sólida reputación ligada a la innovación y a la calidad educativa.

Otro hecho que justifica la importancia de la valoración realizada sobre la marca es el fuerte grado de orientación hacia el estudiante -consumidor-que impregna la cultura organizativa de esta universidad. Se trata de 
una institución de educación a distancia, por lo que su modelo educativo y de gestión está pensado para satisfacer las necesidades de un estudiante que, de media, supera los 30 años de edad y que busca conciliar vida laboral y personal con una formación continuada a lo largo de toda la vida. Además, se trata de un estudiante que, mayoritariamente, ejerce una actividad profesional, por lo que busca una formación académica con fuerte enfoque práctico y aplicado.

La capacidad de la universidad para cumplir con las promesas realizadas será clave en el presente de la universidad, ya que de ello dependerán tanto las relaciones que mantiene actualmente con sus estudiantes como la capacidad para atraer a nuevos estudiantes. $Y$ es que se trata de un estudiante muy exigente e informado, con poder adquisitivo, pero que, a la vez, presenta limitaciones de tiempo. Es por ello que la credibilidad de la universidad será fundamental en la valoración que el estudiante realice de la marca de la universidad, y, con ello, en la experiencia que obtenga y en los niveles de fidelidad mostrados.

La calidad del sistema, así como la relación que el estudiante mantiene a través del campus virtual, aparecen como otros dos elementos fundamentales en la experiencia del estudiante con la universidad online. Trabajos como los de Park (2009) o Schneckenberg (2010), entre otros, señalan la existencia de tres importantes barreras a la hora de que un estudiante inicie sus estudios a distancia en la modalidad online. Las dos primeras, vinculadas con la tecnología que le da soporte a la obtención del servicio, como son la facilidad de uso y la utilidad percibida, mientras que la tercera está relacionada con la sensación de aislamiento o soledad que acompaña al estudiante durante sus horas de estudio.

De forma particular, el estudiante que apuesta por el e-learning ha de poseer determinadas competencias tecnológicas. Pero aquellas que son inherentes a un nativo digital en ocasiones constituyen todo un reto para un emigrante digital. Es por ello que el estudiante que, bajo estas condiciones, decide seguir su formación superior en una universidad online ha de ser consciente de que ha de adoptar una tecnología con mayor o menor grado de facilidad de uso y utilidad percibida.

El estudiante mejorará su experiencia cuando la tecnología que da soporte al aula virtual permite la navegación y el acceso a los contenidos de forma fácil, rápida e intuitiva, o cuando hace posible que el proceso de adquisición de conocimientos y competencias se realice de forma práctica, divertida y colaborativa. Asimismo, la disponibilidad de acceder a recursos multimedia externos, o hacer que la comunicación con otros miembros de la comunidad universitaria sea interactiva, también incrementa la experiencia del estudiante. Por otro lado, el acompañamiento constate es vital para reducir la sensación de soledad y aislamiento que sufre el estudiante online. El estudiante necesita saber que no está solo y que puede contar con la ayuda tanto de docentes como de tutores. $Y$ en este sentido, la experiencia del estudiante será mejor a medida que el staff académico demuestre una elevada experiencia académica y profesional en el ámbito del conocimiento objeto de estudio, así como también cuando estos profesionales sean capaces de empatizar con el estudiante, entender sus problemas y ayudarle a resolver situaciones complejas.

La universidad virtual es consciente de esta situación $y$, por ello, desde un principio ha realizado una fuerte apuesta por la renovación constante del entorno web. Con la incorporación permanente de herramientas 2.0, permite que, en el entorno online, la relación entre el estudiante y el staff académico (profesores-consultores y profesores-tutores) sea próxima y directa. Pero, junto a la incidencia directa que estos elementos tienen sobre la experiencia del estudiante, también se debe considerar que, de una manera indirecta, inciden en la imagen de la marca de la universidad, y en la valoración que de la misma realizan los estudiantes, consolidando de esta forma una imagen de universidad innovadora, pero, a la vez, próxima y abierta.

Finalmente, cabe señalar que la calidad de la información y, en concreto, de los recursos y materiales docentes utilizados no se muestra como un elemento determinante en la experiencia del estudiante con la universidad. En este sentido, es importante puntualizar que para un estudiante joven, que inicia su primer contacto con la universidad, la calidad de los materiales docentes es fundamental en su proceso de aprendizaje. Pero cuando el estudiante dispone de conocimientos y experiencia laboral en el área de conocimiento y, además, desarrolla el proceso de aprendizaje en un contexto online, la importancia de los recursos y materiales docentes se reduce. El estudiante es consciente de sus necesidades y, por ello, es capaz de juzgar la calidad de los recursos e incluso de buscar recursos actualizados en la red. 


\section{BIBLIOGRAFÍA}

Aaker, D. [1991]: Building strong brands, New York: The Free Press.

Akbar, A. y Mannan, A. [2015]: «The role of online service quality in enhancing customer satisfaction: an empirical investigation of pakistani banks», International Journal of Information, Business and Management, 7 (4), págs. 272-283.

Asthana, A. [19th November 2006]: «Angry students demand value for fee money», The Guardian. Disponible en: https://www.theguardian.com/uk/2006/nov/19/ highereducation.students [Consultado: septiembre de 2017].

Balaji, M. S.; Roy, S. K. y Sadeque, S. [2016]: «Antecedents and consequences of university brand identification», Journal of Business Research, 69 (8), págs. 3.023-3.032. DOI 10.1016/j.jbusres.2016.01.017.

Balmer, J. M. T. y Liao, M.-N. [2007]: «Student corporate brand identification: an exploratory case study», Corporate Communications: An International Journal, 12 (4), págs. 356-375.

Bharati, P. y Chaudhury, A. [2004]. «An empirical investigation of decision-making satisfaction in web-based decision support systems», Decision Support Systems, 37 (2), págs.187-197.

Bigné-Alcañiz, J. E. y Andreu-Simó, L. [2004]: «Modelo cognitivo-afectivo de la satisfacción en servicios de ocio y turismo», Cuadernos de Economía y Dirección de la Empresa, 21, págs. 89-120.

Bishop, D. [22-24 June 2015]: «Conceptualising the student-university relationship within a UK higher education institute», 5th Cambridge Student Voice Seminar, University of Cambridge.

Blackston, M. y Lebar, E. [2015]: «Constructing consumer-brand relationships to better market and build businesses», en S. Fournier, M. J. Breazeale y J. Avery (eds.), Strong brands, strong relationships, Abingdon (Oxford): Routledge, pág. 376.

Brakus, J. J.; Schmitt, B. H. y Zarantonello, L. [2009]: «Brand experience: What is it? How is it measured? Does it affect loyalty?», Journal of Marketing, 73 (3), págs. 52-68.

Cabero Almenara, J. y Llorente Cejudo, M. ${ }^{a}$ C. [2009]: «Actitudes, satisfacción, rendimiento académico y co- municación online en procesos de formación universitaria en blended leaning», Teoría de la Educación: Educación y Cultura en la Sociedad de la Información, 10 (1), págs. 172-189.

Carbone, L. P. y Haeckel, S. H [1994]: «Engineering customer experiences», Marketing Management, 3 (3), págs. 9-11.

Chamde Farias, S. A.; Aguiar, E. C. y Melo, F. V. S. [2014]: «Store atmospherics and experiential marketing: a conceptual framework and research propositions for an extraordinary customer experience», International Business Research, 7(2), págs. 87.

Chang, H. H. y Chen, S. W. [2008]: «The impact of online store environment cues on purchase intention: trust and perceived risk as a mediator», Online Information Review, 32 (6), págs. 818-841.

Chang, K.-C.; Kuo, N.-T.; Hsu, C.-L. y Cheng, Y.-S. [2014]: «The impact of website quality and perceived trust on customer purchase intention in the hotel sector: website brand and perceived value as moderators", International Journal of Innovation, Management and Technology, 5 (4), págs. 255-260.

Chernatony, L. de y McDonald, M. [1998]: Creating powerful brands in consumer, services and industrial markets, 2nd ed., Oxford: Butterworth-Heinemann.

Chong, Y. S. y Ahmed, P. K. [2015]: «Student motivation and the "feel good" factor: an empirical examination of motivational predictors of university service quality evaluation», Studies in Higher Education, 40 (1), págs. 158-177.

Cova, B. y Dalli, D. [2009]: «Working consumers: the next step in marketing theory?», Marketing Theory, 9 (3), págs. 315-339.

D'Este, P.; Castro Martínez, E. y Molas-Gallart, J. [2009]: Documento de base para un manual de indicadores de vinculación de la universidad con el entorno socioeconómico: un marco para la discusión, Observatorio Iberoamericano de la Ciencia, la Tecnología y la Sociedad, INGENIO (CSIC-UPV), España.

Dabholkar, P. A. y Sheng, X. [2012]: «Consumer participation in using online recommendation agents: effects on satisfaction, trust, and purchase intentions», The Service Industries Journal, 32 (9), págs. 1.433-1.449. 
DeLone, W. H. y McLean, E. R. [2003]. «The DeLone and McLean model of information», Journal of Management Information Systems, 19 (4), págs. 9-30.

Dennis, C.; Papagiannidis, S.; Alamanos, E. y Bourlakis, M. [2017]: «The role of brand attachment and its antecedents in brand equity in higher education», en M. Stieler (ed.), Creating marketing magic and innovative future marketing trends, Springer, págs. 287-292.

Elsharnouby, T. [2015]: «Student co-creation behavior in higher education: the role of satisfaction with the university experience», Journal of Marketing for Higher Education, 25 (2), págs. 238-262.

Erdem, T. y Swait, J. [1998]: «Brand equity as a signaling phenomenon», Journal of Consumer Psychology, 7 (2), págs. 131-157.

Esch, F. R; Möll, T.; Schmitt, B.; Elger, C. E.; Neuhaus, C. y Weber, B. [2012]: «Brands on the brain: Do consumers use declarative information or experienced emotions to evaluate brands?», Journal of Consumer Psychology, 22, págs. 75-85.

Falk, R. F. y Miller, N. B. [1992]: A primer for soft modeling, Ohio (EE. UU.): University of Akron Press.

Farias, S. A. de; Aguiar, E. C. y Sales Melo, F. V. [2014]: «Store atmospherics and experiential marketing: a conceptual framework and research propositions for an extraordinary customer experience», International Business Research, 7 (2), págs. 87-99.

Farrell, A. M.; Souchon, A. L. y Durden, G. R. [2001]: «Service encounter conceptualisation: employees' service behaviours and customers' service quality perceptions», Journal of Marketing Management, 17, págs. 577-593.

Fornell, C. y Larcker, D. F. [1981]. «Evaluating structural equation models with unobservable variables and measurement error», Journal of Marketing Research, 18 (1), págs. 39-50.

Frow, P. y Payne, S. A. [2007]. «Towards the perfect customer experience», Journal of Brand Management, 15 (2), págs 89-101.

Gentile, C.; Spiller, N. y Noci, G. [2007]: «How to sustain the customer experience: an overview of experience components that co-create value with the customer», European Management Journal, 25 (5), págs. 395-410.
Goldhaber, D. D. y Eide, E. R. [2003]: «Methodological thoughts on measuring the impact of private sector competition on the educational marketplace», Educational Evaluation and Policy Analysis, 25 (2), págs. 217-232.

Gómez-Suárez, M.; Martínez-Ruiz, M. ${ }^{a}$ P. y MartínezCaraballo, N. [22 February 2017]: «Consumer-brand relationships under the marketing 3.0 paradigm: a literature review», Frontiers in Psychology. DOI https:// doi.org/10.3389/fpsyg.2017.00252.

Grace, D. y O'Cass, A. [2004]: «Examining service experiences and post-consumption evaluations», Journal of Services Marketing, 18 (6), págs.450-461.

Greenley, G. E.; Hooley, G. J. y Rudd, J. M. [2005]: «Market orientation in a multiple stakeholder orientation context: implications for marketing capabilities and assets», Journal of Business Research, 58 (11), págs. 1.483-1.494.

Hair, J. F.; Anderson, R. E.; Tatham, R. L. y Black, W. C. [1999]: «Análisis multivariante», ${ }^{\text {a }}{ }^{\text {ed., Madrid: Pren- }}$ tice Hall Iberia.

Hartono, E.; Holsapple, C. W.; Kim, K.-Y.; Na, K.-S. y Simpson, J. T. [2014]: «Measuring perceived security in B2C electronic commerce website usage: a respecification and validation», Decision Support Systems, 62, págs. 11-21.

Hsieh, S. H. y Chang, A. [2016]: «The psychological mechanism of brand co-creation engagement», Journal of Interactive Marketing, 33, págs. 13-26.

Janda, S.; Trocchia, P. J. y Gwinner, K. P. [2002]: «Consumer perceptions of internet retail service quality", International Journal of Service Industry Management, 13 (5), págs. 412-431.

Jöreskog, K. y Sörbom, D. [1993]: LISREL 8: structural equation modeling with the SIMPLIS command language, Hillsdale (New Jersey, EE. UU.): Lawrence Erlbaum Associates Publishers.

Kauffman, H. [2015]: «A review of predictive factors of student success in and satisfaction with online learning», Research in Learning Technology, 23 (1).

Kelley, S. W.; Donnelly, J. H. Jr. y Skinner, S. J. [1990]: "Customer participation in service production and delivery», Journal of Retailing, 66 (3), págs. 315-335.

Kumar, V.; Dalla Pozza, I. y Ganesh, J. [2013]: «Revisiting the satisfaction-loyalty relationship: empirical generalizations and directions for future research», Journal of Retailing, 89 (3), págs. 246-262. 
Lasalle, D. y Britton, T. A. [2003]: Priceless: turning ordinary products into extraordinary experiences, Boston (EE. UU.): Harvard Business School Press.

Lemke, F.; Clark, M. y Wilson, H. [2011]: «Customer experience quality: an exploration in business and consumer contexts using repertory grid technique», Journal of the Academy of Marketing Science, 39 (6), págs. 846-869.

Lemon, K. N. y Verhoef, P. C. [2016]: «Understanding customer experience throughout the customer journey», Journal of Marketing, 80 (6), págs. 69-96.

Leverin, A. y Liljander, V. [2006]: «Does relationship marketing improve customer relationship satisfaction and loyalty?», International Journal of Bank Marketing, 24 (4), págs. 232-251.

Lin, H.-F. [2007]: «The impact of website quality dimensions on customer satisfaction in the B2C e-commerce context», Total Quality Management \& Business Excellence, 18 (4), págs 363-378.

Lowrie, A. [2007]: «Branding higher education: equivalence and difference in developing identity», Journal of Business Research, 60 (9), págs. 990-999.

Marconi, J. [1993]: Beyond branding: how savvy marketers build brand equity to create products and open new markets, Chicago (IL): Probus Publishing Company.

Mateo, A. [2015]: «Recapturing marketing from the marketization of higher education discourse», 3rd Mediterranean Interdisciplinary Forum on Social Science and Humanities, MIFS 2015, 17-19 de mayo, Barcelona (España), págs. 21-36.

Meyer, C. y Schwager, A. [2007]: «Understanding customer experience», Harvard Business Review, 85 (2), 116-126.

Morgan, M.; Lugosi, P. y Brent Ritchie, J. R. (eds.) [2010]: The tourism and leisure experience: consumer and managerial perspectives, United Kingdom: Channel View Publications.

Munteanu, C.; Ceobanu, C.; Bobâlcă, C. y Anton, O. [2010]: «An analysis of customer satisfaction in a higher education context», International Journal of Public Sector Management, 23 (2), págs. 124-140.

$\mathrm{Ng}$, I. C. L. y Forbes, J. [2009]: «Education as service: the understanding of university experience through the service logic», Journal of Marketing for Higher Education, 19 (1), págs. 38-64.

Nunnally, J. C. [1978]: Psychometric theory, 2. ${ }^{a}$ ed., New York (EE. UU.): McGraw-Hill.

Palmer, J. W. [2002]: «Web site usability, design, and performance metrics», Information Systems Research, 13 (2), págs. 151-167.

Pansari, A. y Kumar, V. [2017]: «Customer engagement: the construct, antecedents, and consequences», Journal of the Academy of Marketing Science, 45 (3), págs. 294-311.

Parasuraman, A.; Zeithaml, V. A. y Berry, L. L. [1985]: «A conceptual model of service quality and its implications for future research», Journal of Retailing, 49, págs. 44-60.

[1988]: «SERVQUAL: a multiple-item scale for measuring consumer perceptions of service quality», Journal of Retailing, 64 (1), págs. 12-40.

Park, S. Y. [2009]: «An analysis of the technology acceptance model in understanding university students' behavioral intention to use e-learning», Journal of Educational Technology \& Society, 12 (3), págs. 150.

Patlán-Pérez, J.; Martínez, E. y Hernández, R. [2012]: «El clima y la justicia organizacional y su efecto en la satisfacción laboral», Revista Internacional Administración \& Finanzas, 5 (5), págs. 1-19.

Pimienta, M. ${ }^{a}$ L.; Barzola, M. ${ }^{a}$ L. y Zurdo, F. H. [2016]: «La imagen de las universidades en Mendoza: percepciones, expectativas y significación de su rol actual», Diálogos Pedagógicos, 13 (26), págs. 62-87.

Polat, F. [2011]: «Inclusion in education: a step towards social justice», International Journal of Development, 31 (1), págs. 50-58.

Propper, C.; Wilson, D. y Burgess, S. [2006]: «Extending choice in english health care: the implications of the economic evidence», Journal of Social Policy, 35 (4), págs. 537-557.

Ringle, C. M.; Wende, S. y Will, A. [2005]: SmartPLS 2.0 (beta), Hamburgo (Alemania): University of Hamburg. Disponible en: http:// www.smartpls.de. [Consultado: septiembre de 2017].

Rose, S.; Clark, M.; Samouel, P. y Hair, N. [2012]: «Online customer experience in e-retailing: an empirical 
model of antecedents and outcomes», Journal of Retailing, 88 (2), págs. 308-322.

Schmitt, B. H. [2000]: Experiential marketing: how to get customers to sense, feel, think, act, relate, Simon and Schuster. The Free Press, New York.

Schmitt, B. y Zarantonello, L. [2013]: «Consumer experience and experiential marketing: a critical review», Review of Marketing Research, 10, págs. 25-61.

Schneckenberg, D. [2010]: "Overcoming barriers for elearning in universities-portfolio models for ecompetence development of faculty", British Journal of Educational Technology, 41 (6), págs. 979-991.

Shapiro, H. B.; Lee, C. H.; Roth, N. E. W.; Li, K.; Çetinkaya-Rundel, M. y Canelas, D. A. [2017]: «Understanding the massive open online course (MOOC) student experience: an examination of attitudes, motivations, and barriers», Computers \& Education, 110 , págs. $35-50$.

Sharma, N. y Patterson, P. G. [2000]: «Switching costs, alternative attractiveness and experience as moderators of relationship commitment in professional, consumer services», International Journal of Service Industry Management, 11 (5), págs. 470-490.

Shaw, C. e Ivens, J. [2005]: Building great customer experiences, Londres (Reino Unido): Prentice-Hall.

Smilansky, S. [2009]: Experiential marketing: a practical guide to interactive brand experiences, EE. UU: Kogan Page Publishers.

Srivastava, M. y Kaul, D. [July 2016]: «Exploring the link between customer experience-loyalty-consumer spend», Journal of Retail and Consumer Services, 31, págs. 277-286.

Steenkamp, J.-B. y Trijp, H. C. van [1991]: «The use of LISREL in validating marketing constructs», International Journal of Research in Marketing, 8, págs. 283-299.

Traverso Cortes, J. [2005]: «lmagen interna de la institución universitaria. Modelo para el personal de administración y servicios», Revista de Economía y Empresa, XXIII (54/55), págs. 95-112.
Turley, L. W. y Moore, P. A. [1995]: «Brand name strategies in the service sector», The Journal of Consumer Marketing, 12 (4), págs. 42-50.

Tynan, C. y McKechnie, S. [2009]: «Experience marketing: a review and reassessment», Journal of Marketing Management, 25 (5-6), págs. 501-517.

Vargo, S. L. y Lusch, R. F. [2008]: «Service-dominant logic: continuing the evolution», Journal of the Academy of Marketing Science, 36 (1), págs. 1-10.

Vijayasarathy, L. R. [2004]: «Predicting consumer intentions to use on-line shopping: the case for an augmented technology acceptance model», Information \& Management, 41, págs. 747-762.

Voss, C. y Zomerdijk, L. [2007]: Innovation in experiential services-an empirical view, London (United Kingdom): Advanced Institute of Management Research/London Business School.

Wei, W.; Torres, E. y Hua, N. [2016]: Improving consumer commitment through the integration of self-service technologies: a transcendent consumer experience perspective. International Journal of Hospitality Management, 59, págs. 105-115.

Williams, R. L. Jr. y Omar, M. [2014]: «Applying brand management to higher education through the use of the brand flux model-the case of Acadia University», Journal of Marketing for Higher Education 24 (2), págs. 222-242.

Wu, Y. C. J.; Shen, J. P. y Chang, C. L. [2015]: «Electronic service quality of Facebook social commerce and collaborative learning», Computers in Human Behavior, 51, págs. 1.395-1.402.

Xiao, J. y Wilkins, S. [2015]: «The effects of lecturer commitment on student perceptions of teaching quality and student satisfaction in chinese higher education», Journal of Higher Education Policy and Management, 37 (1), págs. 98-110.

Zomerdijk, L. G. y Voss, C. A. [2011]: «NSD processes and practices in experiential services», Journal of Product Innovation Management, 28, págs. 68-80. 


\section{APÉNDICES}

\section{Variables empleadas en el estudio}

\begin{tabular}{|c|c|}
\hline Variable & Dimensión \\
\hline $\begin{array}{l}\text { - Valoración de los recursos de aprendizaje y de los materiales docentes. } \\
\text { - Valoración del aula virtual. } \\
\text { - Valoración de la comunicación en el aula virtual. } \\
\text { - Valoración de los medios y recursos comunicativos del aula (chats, wikis y foros). } \\
\text { - Valoración de los servicios de atención al estudiante. }\end{array}$ & Calidad del sistema \\
\hline $\begin{array}{l}\text { - Organización de los materiales y recursos de aprendizaje. } \\
\text { - Influencia de los recursos de aprendizaje en la asimilación del conocimiento. } \\
\text { - Nivel de actualización de los recursos de aprendizaje. } \\
\text { - Posibilidad de usar fuentes de información externas adicionales en el propio espacio de recursos del aula. } \\
\text { - Nivel de ajuste de la información ofrecida a las necesidades del estudiante. } \\
\text { - La información es tratada con seriedad. }\end{array}$ & $\begin{array}{l}\text { Calidad de la } \\
\text { información }\end{array}$ \\
\hline $\begin{array}{l}\text { Profesor-tutor }\left\{\begin{array}{l}\text { El profesor-tutor me orienta de forma adecuada. } \\
\text { E El profesor-tutor responde con claridad. } \\
\cdot \text { El profesor-tutor/acompaña al estudiante. } \\
\cdot \text { El profesor-tutor me motiva a mantener el ritmo de estudio. }\end{array}\right. \\
\text { Profesor-consultor }\left\{\begin{array}{l}\cdot \text { El profesor-consultor domina la asignatura. } \\
\cdot \text { El profesor-consultor ha planificado adecuadamente el aprendizaje. } \\
\cdot \text { El profesor-consultor responde en el término adecuado. } \\
\cdot \text { El profesor-consultor ofrece un trato personalizado. }\end{array}\right.\end{array}$ & Calidad del servicio \\
\hline $\begin{array}{l}\text { - La UOC mejora mis competencias en entornos virtuales. } \\
\text { - Valoro positivamente el título ofrecido por la UOC. } \\
\text { - La UOC me permite relacionarme con personas de mi ámbito. } \\
\text { - Me siento miembro de la comunidad UOC. }\end{array}$ & $\begin{array}{c}\text { Valoración marca } \\
\text { universidad }\end{array}$ \\
\hline & nte: elaboración propia \\
\hline
\end{tabular}




\section{Validación de escalas}

\begin{tabular}{|c|c|c|c|c|c|c|c|}
\hline Factor & Dimensión & Indicador & Carga & Valor $\mathrm{t}$ & $\begin{array}{l}\text { Alfa de } \\
\text { Cronbach }\end{array}$ & $\begin{array}{l}\text { Fiabilidad } \\
\text { compuesta }\end{array}$ & AVE \\
\hline \multirow{2}{*}{$\begin{array}{l}\text { Calidad de la } \\
\text { información }\end{array}$} & INFOR1 & $\begin{array}{l}\text { INFORMATI1 } \\
\text { INFORMATI2 } \\
\text { INFORMATI3 }\end{array}$ & $\begin{array}{l}, 79 \\
, 89 \\
, 89\end{array}$ & $\begin{array}{l}16,01 \\
17,03 \\
17,12\end{array}$ & \multirow[t]{2}{*}{833} & \multirow[t]{2}{*}{,921 } & \multirow[t]{2}{*}{, 704} \\
\hline & INFOR2 & $\begin{array}{l}\text { SECURI1 } \\
\text { SECURI2 }\end{array}$ & $\begin{array}{l}, 99 \\
, 58\end{array}$ & $\begin{array}{c}24,89 \\
6,47\end{array}$ & & & \\
\hline \multirow[t]{2}{*}{ Calidad del sistema } & SIST1 & $\begin{array}{l}\text { WEBDISG1 } \\
\text { WEBDISG2 } \\
\text { WEBDISG3 } \\
\text { WEBDISG4 }\end{array}$ & $\begin{array}{l}, 85 \\
, 84 \\
, 96 \\
, 85\end{array}$ & $\begin{array}{l}18,24 \\
18,67 \\
23,88 \\
19,35\end{array}$ & \multirow[t]{2}{*}{,938 } & \multirow[t]{2}{*}{0,960} & \multirow[t]{2}{*}{0,800} \\
\hline & SIST2 & $\begin{array}{l}\text { INTERACT1 } \\
\text { INTERACT2 }\end{array}$ & $\begin{array}{l}, 93 \\
, 93\end{array}$ & $\begin{array}{l}25,63 \\
25,28\end{array}$ & & & \\
\hline \multirow{3}{*}{$\begin{array}{l}\text { Calidad servicio } \\
\text { tutor }\end{array}$} & SERTUT1 & $\begin{array}{l}\text { RESPONS1 } \\
\text { RESPONS2 }\end{array}$ & $\begin{array}{l}, 98 \\
, 89\end{array}$ & $\begin{array}{l}16,71 \\
18,49\end{array}$ & \multirow{3}{*}{,931 } & \multirow{3}{*}{0,960} & \multirow{3}{*}{0,778} \\
\hline & SERTUT2 & $\begin{array}{l}\text { TRUST1 } \\
\text { TRUST2 }\end{array}$ & $\begin{array}{l}, 88 \\
, 92\end{array}$ & $\begin{array}{l}17,54 \\
20,62\end{array}$ & & & \\
\hline & SERTUT3 & $\begin{array}{l}\text { EMPATH1 } \\
\text { EMPATH2 } \\
\text { EMPATH3 }\end{array}$ & $\begin{array}{l}, 93 \\
, 82 \\
, 73\end{array}$ & $\begin{array}{l}22,12 \\
20,20 \\
15,32\end{array}$ & & & \\
\hline \multirow[t]{2}{*}{$\begin{array}{l}\text { Calidad servicio } \\
\text { consultor }\end{array}$} & SERCONS1 & $\begin{array}{c}\text { RESPONS1 } \\
\text { RESPONS2 } \\
\text { EMPATH1 } \\
\text { EMPATH2 }\end{array}$ & $\begin{array}{l}, 71 \\
, 74 \\
, 80 \\
, 91\end{array}$ & $\begin{array}{l}13,84 \\
15,85 \\
12,69 \\
14,32\end{array}$ & \multirow[t]{2}{*}{,923 } & \multirow[t]{2}{*}{0,932} & \multirow[t]{2}{*}{0,696} \\
\hline & SERCONS2 & $\begin{array}{l}\text { TRUST1 } \\
\text { TRUST2 }\end{array}$ & $\begin{array}{l}, 89 \\
, 93\end{array}$ & $\begin{array}{l}15,23 \\
23,62\end{array}$ & & & \\
\hline Marca universidad & BRAND & $\begin{array}{l}\text { BRAND1 } \\
\text { BRAND2 } \\
\text { BRAND3 } \\
\text { BRAND4 }\end{array}$ & $\begin{array}{l}, 68 \\
, 72 \\
, 80 \\
, 78\end{array}$ & $\begin{array}{l}12,11 \\
12,90 \\
14,86 \\
14,54\end{array}$ & ,842 & 0,834 & 0,557 \\
\hline & & & & & & \multicolumn{2}{|c|}{ Fuente: elaboración propi } \\
\hline
\end{tabular}




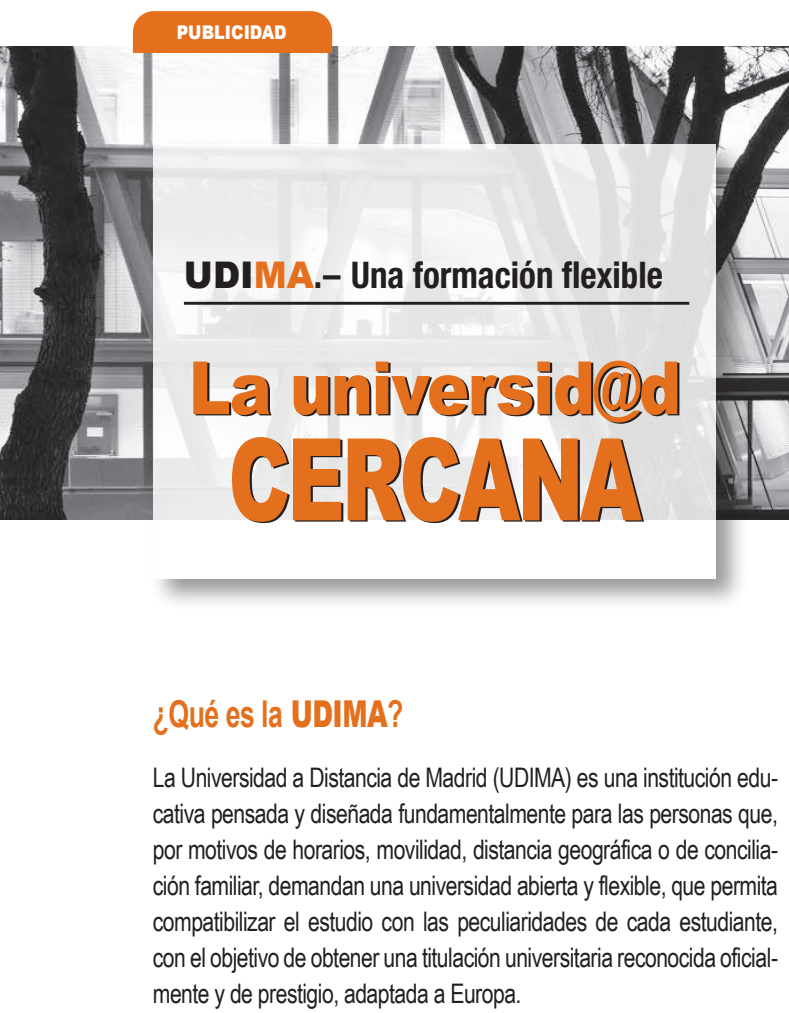

\section{¿Cómo se adapta a cada estudiante?}

Ofreciendo un acceso sencillo y permanente a las aulas virtuales, sin restricciones de horarios, todos los días del semestre académico.

Asesorando a cada estudiante de forma personalizada, especialmente en el trámite de la matrícula, para escoger las asignaturas que mejor se adapten al tiempo disponible y a la capacidad de cada uno y a través del seguimiento continuo de profesores y tutores.

Planificando el estudio a través de la «Guía docente de la asignatura», de la realización de actividades didácticas y de su entrega, en un sistema de comunicación y evaluación continua, en el que las actividades propuestas están pensadas para la asimilación paulatina de los conocimientos de forma sencilla, comprendiendo la utilidad práctica de los mismos.

\section{Fechas de exámenes}

Los exámenes ordinarios se realizan el último fin de semana de enero y el primero de febrero, y el último de junio y el primero de julio, y el extraordinario, en el primer fin de semana del mes de septiembre.

\section{¿Cómo son los exámenes en la UDIMA y dónde se hacen?}

Los exámenes finales semestrales son presenciales y con carácter obligatorio. Este tipo de prueba de evaluación permite constatar el cumplimiento de los objetivos de aprendizaje previstos en cada asignatura.

Para poder presentarse al examen final será requisito indispensable la realización de las actividades didácticas que se establezcan en la guía docente de cada asignatura.

Sedes de examen: A Coruña, Alicante, Aranda de Duero, Barcelona, Bilbao, Collado Villalba, Córdoba, Las Palmas de Gran Canaria, Madrid, Málaga, Mérida, Oviedo, Palma, Sevilla, Tenerife, Valencia, Vigo y Zaragoza.

Para exámenes en el extranjero consulte: www.udima.es.

\section{Los materiales}

Hemos seleccionado los mejores textos y autores para estudiar cada asignatura. Estos contenidos se complementan con notas técnicas, consultas a bases de datos, bibliotecas digitales, etc.

Todos los materiales que necesita el alumno para desarrollar las distintas asignaturas están incluidos en el precio de los créditos. No hay que realizar ningún desembolso adicional. Para gastos de envío fuera de España consulte: www.udima.es.

\section{Metodología de estudio}

El proceso enseñanza-aprendizaje se desarrolla a través de las aulas virtuales de la UDIMA. Nuestros estudiantes pueden establecer una comunicación directa con sus profesores a través de los foros, las tutorías telefónicas y las herramientas telemáticas complementarias que permiten la comunicación en tiempo real.

\section{Reconocimiento de créditos (convalidaciones)}

El estudio de reconocimiento de créditos que la UDIMA realiza para determinar las asignaturas que un alumno puede convalidar es gratuito; no obstante, el alumno deberá abonar un $10 \%$ del coste en primera matrícula por cada asignatura que finalmente decida incluir en su expediente. 


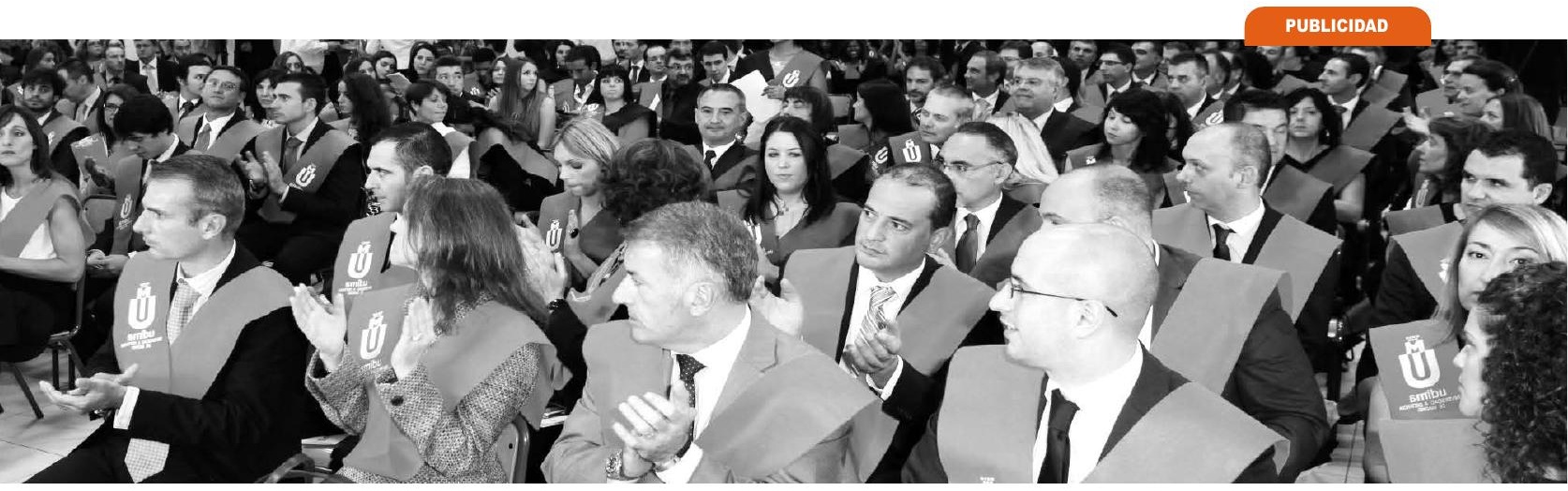

\section{Cómo es el perfil de los alumnos de la UDIMA}

/ Por qué somos tu mejor opción

Personas que tienen en la cabeza la necesidad de formarse

La mayor parte de nuestros alumnos compaginan el trabajo y la vida personal con la formación, porque saben que es la única manera de seguir creciendo.

Personas que saben ver la evolución de la sociedad y la tecnología

Si hoy en día nos enteramos de lo que pasa en el otro lado del mundo de manera inmediata o tenemos reuniones por videoconferencia, ¿por qué no podemos aprovechar la tecnología para estudiar?

\section{Alumnos que demandan comunicación} constante

La tecnología es solamente el medio. El equipo de profesores, tutores personales y asesores académicos que acompañan al alumno en su experiencia formativa es nuestra razón de ser.

\section{Amor propio y coraje}

Nuestros estudiantes nunca se rinden. Saben que el aprendizaje es un proceso en el que van a invertir mucho esfuerzo, pero también saben que la recompensa merece la pena.

\section{Profesionales que tienen en la mano} cambiar su futuro

Gente inconformista, que necesita una universidad que se adapte a su ritmo de vida y que cree en la excelencia formativa. Personas como tú. ¿A qué estás esperando?

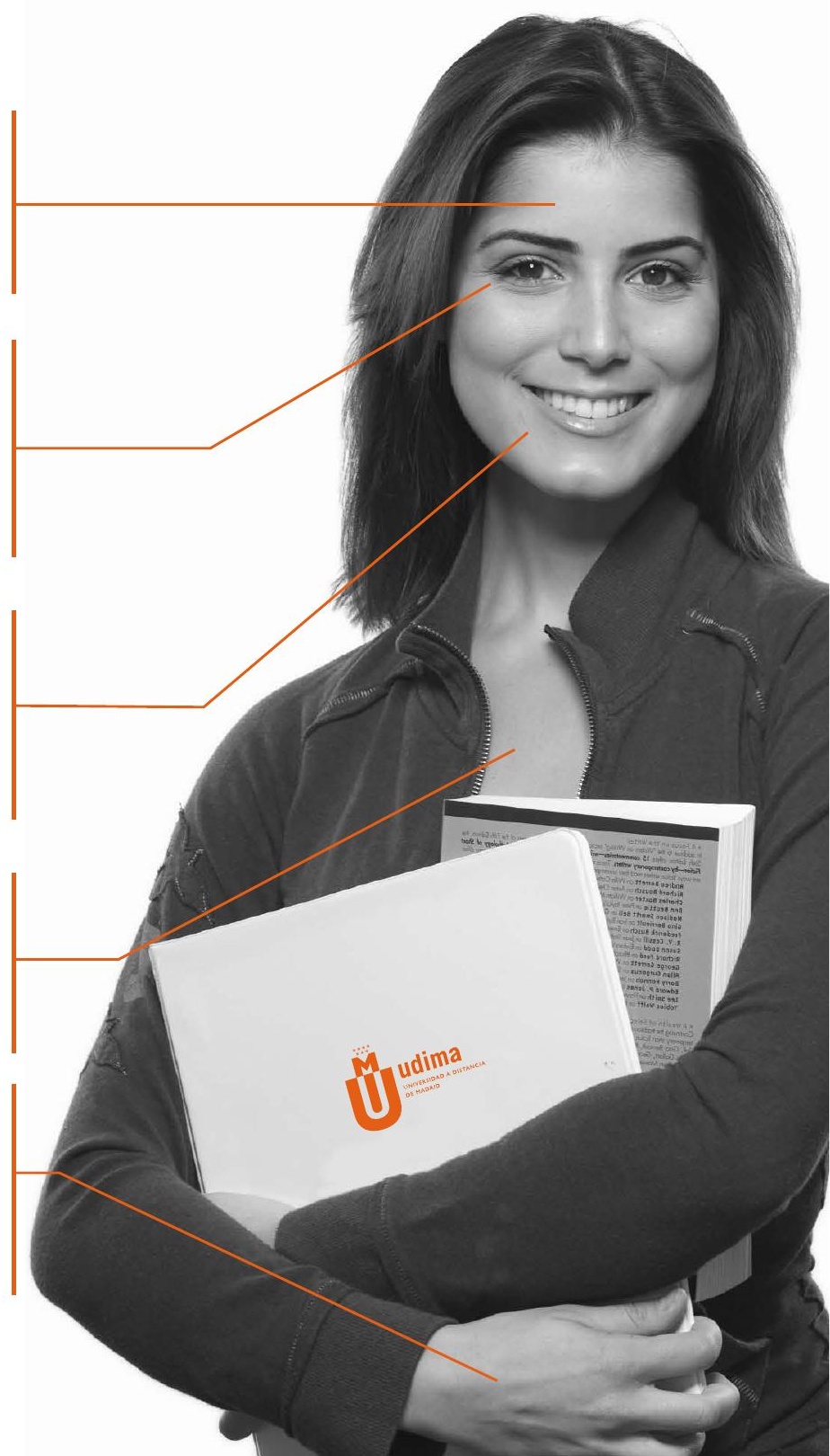



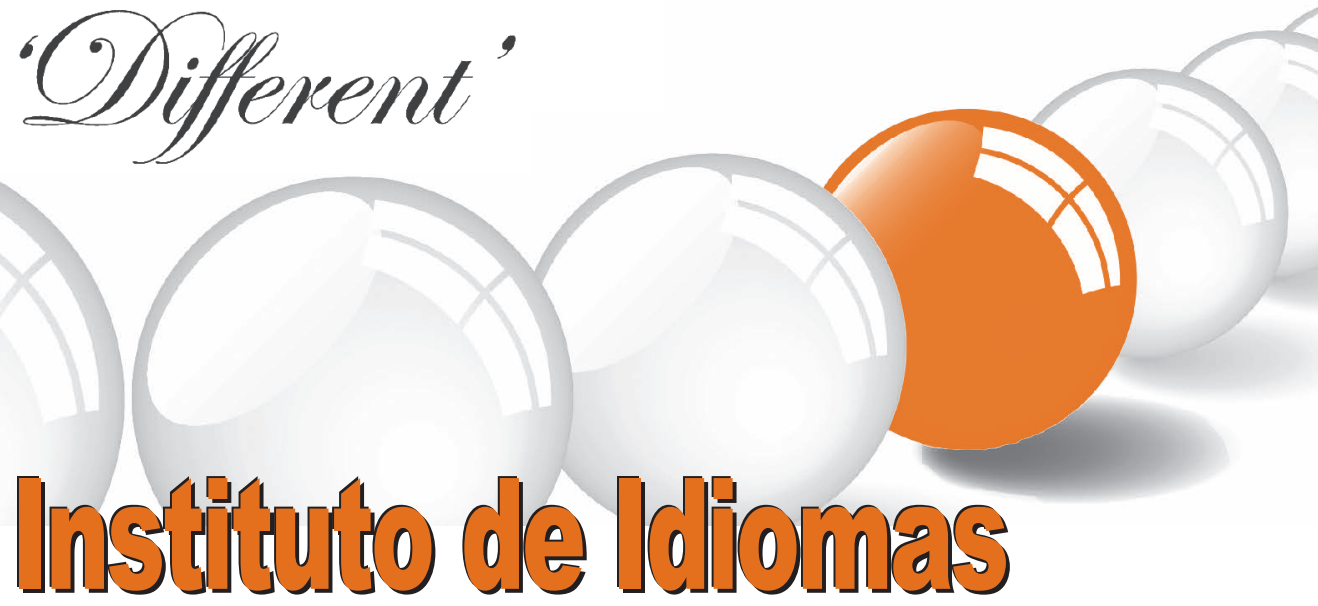

\section{Una enseñanza orientada al mercado laboral}

El Instituto de Idiomas de la UDIMA (IIU), ante la gran necesidad e interés reciente por el aprendizaje de lenguas extranjeras, ofrece a su comunidad universitaria y al público en general una enseñanza de idiomas orientada al mercado laboral.

\section{Metodología personalizada con resultados de aprendizaje garantizados}

La formación en lenguas extranjeras se lleva a cabo dentro de la normativa vigente de la enseñanza de idiomas en España y en la Unión Europea. Todos los cursos presentan un diseño adaptado al Marco Común Europeo de Referencia para las lenguas y a la metodología propia de la UDIMA: un sistema de enseñanza cercano, flexible, actual, dinámico y personalizado.

Los cursos se desarrollan con metodología online aplicada a la enseñanza de idiomas; un sistema de enseñanza basado en la personalización del aprendizaje, la tecnología de vanguardia y la utilización de herramientas que permiten la comunicación en tiempo real con el alumnado.

La enseñanza se imparte por profesores universitarios cualificados en idiomas extranjeros y expertos en la enseñanza de idiomas e-learning.

Los alumnos, después de ser evaluados favorablemente, reciben un diploma acreditativo del nivel completo superado (A1.2, A2.2, B1.2, B2.2, C1.2) con los créditos correspondientes reconocidos por el IIU.

\section{Oferta académica adaptada al profesional del siglo XXI}

EI IIU tiene previsto ampliar su oferta formativa y servicios progresivamente. Para ver los cursos que se ofrecen en la actualidad consulte:

\section{El instituto de idiomas te ofrece}

- Un programa formativo que ayuda para la superación de los exámenes de habilitación que algunas comunidades exigen para poder ser profesor de asignaturas de contenidos lingüísticos en inglés en centros concertados bilingües.

- La posibilidad de formarse para obtener el certificado oficial de nivel Cambridge ESOL, al ser centro asociado por el Programa BEDA. Dicha certificación es válida en todas las comunidades para la solicitud de habilitación como profesor de idiomas en primaria o secundaria en centros privados concertados bilingües.

- Certificación BEDA CUM LAUDE, otorgada por Escuelas Católicas de Madrid y la Universidad de Cambridge por la implantación de la docencia universitaria en inglés.

- La posibilidad de examinarse del TOEIC (del que UDIMA es centro autorizado formador y examinador) y obtener la certificación oficial válida para la solicitud de habilitación como profesor de idiomas en primaria o secundaria en centros concertados bilingües.

- Poder examinarse del Oxford Test of English (OTE), ya que es centro formador y examinador, reconocido por ALTE y EALTA como acreditación para los niveles B1 y B2.

- Obtener la certificación Teaching Knowledge Test (TKT) CLIL Module de Cambridge, ya que la formación recibida en el Curso de Experto en Enseñanza Bilingüe (título propio de UDIMA) capacita al candidato para superar con éxito la certificación.

- Formación para la realización de exámenes oficiales de español como lengua extranjera (DELE) del Instituto Cervantes. 